\title{
An Efficient Approach to Dihydrofurocoumarins via Palladium-Catalyzed Annulation of 1,3-Dienes by $o$ - Iodoacetoxycoumarins
}

\author{
Roman V. Rozhkov and Richard C. Larock* \\ Department of Chemistry, Iowa State University, Ames, IA 50011, USA \\ larock@iastate.edu
}

\section{Table of contents}

S1 General, reagents

S2 Annulation of 1,3-dienes by $o$-iodoacetoxycoumarins

S7 Derivatization of dihydrofurocoumarins

S9 References

S10 Scanned ${ }^{1} \mathrm{H}$ and ${ }^{13} \mathrm{C}$ spectra for all compounds prepared

General. All ${ }^{1} \mathrm{H}$ and ${ }^{13} \mathrm{C}$ NMR spectra were recorded at 400 and $100.5 \mathrm{MHz}$ respectively. All melting points are uncorrected. Low resolution mass spectra were recorded on a Finnigan TSQ700 
triple quadripole mass spectrometer (Finnigan MAT, San Jose, CA). High resolution mass spectra were recorded on a Kratos MS50TC double focusing magnetic sector mass spectrometer using EI at 70 eV.

Reagents. Iodine, acetic anhydride, pyridine, mercury(II) acetate, trimethylamine $N$-oxide, 2,3dichloro-5,6-dicyano-p-benzoquinone, osmium tetroxide, 4-hydroxycoumarin, 7-hydroxy-4methylcoumarin, 1,3-cyclohexadiene, 1,3-cycloheptadiene, 1,3-cyclooctadiene, E-1,3-hexadiene, 2,3dimethoxy-1,3-butadiene, 1-methoxy-1,3-cyclohexadiene, 3-methyl-1,3-pentadiene (a mixture of isomers), 2,4-dimethyl-1,3-pentadiene, 2,3-dimethyl-1,3-butadiene, isoprene, 2,4-hexadiene (a mixture of isomers) and 2,3-diphenyl-1,3-butadiene were obtained from commercial sources. E-2-Methyl-1,3pentadiene was purchased from Lancaster Co., Inc. trans,trans-2,4-Hexadiene (95\% pure) was purchased from ChemSamp Co., Inc. E-1-Phenyl-1,3-butadiene and 1,5,5-trimethyl-3methylenecyclohexene were prepared by Wittig condensation according to a literature procedure. ${ }^{1} 5$ Hydroxy-4-methylcoumarin and 6-hydroxycoumarin were prepared as described by Harayama et al. ${ }^{2}$ 4,8-Dimethyl-7-hydroxycoumarin was synthesized by a Pechman condensation. ${ }^{3}$ The preparation and characterization data for compounds 11, 27, 35 and 37 can be found in the Supporting Information for our earlier communication. ${ }^{4}$

\section{General procedure for the Pd-catalyzed annulation of 1,3-dienes by o-iodoacetoxycoumarins.}

The $o$-iodoacetoxycoumarin $(0.25 \mathrm{mmol}), \mathrm{Pd}(\mathrm{dba})_{2}(5 \mathrm{~mol} \%, 0.0125 \mathrm{mmol})$, dppe $(5 \mathrm{~mol} \%, 0.0125$ $\mathrm{mmol}), \mathrm{Ag}_{2} \mathrm{CO}_{3}(0.5 \mathrm{mmol})$ and 1,4-dioxane $(4 \mathrm{~mL})$ were stirred in a capped vial for $5 \mathrm{~min}$, and then water $(1 \mathrm{~mL})$ and the 1,3 -diene $(1.0 \mathrm{mmol})$ were added. The resulting reaction mixture was stirred at $100{ }^{\circ} \mathrm{C}$ for $24 \mathrm{~h}$, cooled to room temperature, filtered and the filtrate was concentrated to give a yellow residue. The resulting residue was purified by column chromatography using silica gel as a solid phase and 4:1 hexanes/ethyl acetate as the eluent to afford after solvent removal the final product. Solid products were then recrystallized from 1:1 ethanol/water. Characterization data for compounds 
9, 16, 17, 21, 23, 24, 32, 36 and 38 can be found in the Supporting Information for our earlier communication. ${ }^{4}$ The following new compounds were prepared using this procedure.

7a,10,11,12,12a-Pentahydro-4-methylcyclohepta[b]-2H-furo[2,3-h]-1-benzopyran-2-one

(14).

Obtained in a $25 \%$ yield: white solid, mp $154-158{ }^{\circ} \mathrm{C} ;{ }^{1} \mathrm{H} \mathrm{NMR}\left(\mathrm{CDCl}_{3}\right)$ 1.53-2.34 (m, 6H), $2.39(\mathrm{~d}$, $J=1.2 \mathrm{~Hz}, 3 \mathrm{H}), 3.77(\mathrm{ddd}, J=10.3,9.1,2.8 \mathrm{~Hz}, 1 \mathrm{H}), 5.60(\mathrm{dd}, J=9.1,1.8 \mathrm{~Hz}, 1 \mathrm{H}), 5.80-5.84(\mathrm{~m}$, $2 \mathrm{H}), 6.10(\mathrm{~d}, J=1.2 \mathrm{~Hz}, 1 \mathrm{H}), 6.75(\mathrm{~d}, J=8.6 \mathrm{~Hz}, 1 \mathrm{H}), 7.41(\mathrm{~d}, J=8.6 \mathrm{~Hz}, 1 \mathrm{H}) ;{ }^{13} \mathrm{C} \mathrm{NMR}\left(\mathrm{CDCl}_{3}\right)$ $19.3,22.4,26.7,26.8,42.3,87.2,106.8,111.4,114.3,118.7,125.7,127.1,129.9,151.1,153.4,161.4$, 162.4; ; IR (neat) 1725, $1605 \mathrm{~cm}^{-1}$; HRMS m/z 268.1102 (calcd for $\mathrm{C}_{17} \mathrm{H}_{16} \mathrm{O}_{3}, 268.1099$ ).

7a,10,11,12,13,13a-Hexahydro-4-methylcycloocta[b]-2H-furo[2,3- $h]$-1-benzopyran-2-one (15). Obtained in a $28 \%$ yield: white solid, mp 126-130 ${ }^{\circ} \mathrm{C} ;{ }^{1} \mathrm{H} \mathrm{NMR}\left(\mathrm{CDCl}_{3}\right)$ 1.18-2.30 (m, 8H), $2.38(\mathrm{~d}$, $J=1.2 \mathrm{~Hz}, 3 \mathrm{H}), 2.78(\mathrm{~m}, 1 \mathrm{H}), 3.85(\mathrm{dt}, J=10.1,2.3 \mathrm{~Hz}, 1 \mathrm{H}), 5.40(\mathrm{ddt}, J=11.8,5.1,1.4 \mathrm{~Hz}, 1 \mathrm{H})$, 5.75-5.95 (m, 2H), $6.09(\mathrm{~d}, J=1.2 \mathrm{~Hz}, 1 \mathrm{H}), 6.78(\mathrm{~d}, J=8.4 \mathrm{~Hz}, 1 \mathrm{H}), 7.40(\mathrm{~d}, J=8.4 \mathrm{~Hz}, 1 \mathrm{H}) ;{ }^{13} \mathrm{C}$ $\operatorname{NMR}\left(\mathrm{CDCl}_{3}\right) \quad 19.4,25.6,26.4,29.7,30.4,47.9,86.0,107.1,111.4,114.5,117.1,125.7,127.9$,

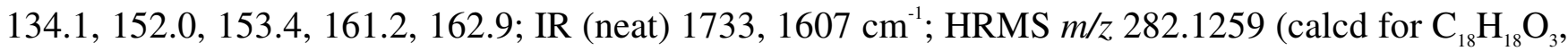
282.1256).

8-(E-1-Butenyl)-8,9-dihydro-4-methyl-2H-furo[2,3-h]-1-benzopyran-2-one (18). Obtained in an 80\% yield: white solid, mp 142-146 ${ }^{\circ} \mathrm{C} ;{ }^{1} \mathrm{H}$ NMR $\left(\mathrm{CDCl}_{3}\right) \quad 1.04(\mathrm{t}, J=7.6 \mathrm{~Hz}, 3 \mathrm{H}), 2.08-2.18(\mathrm{~m}, 2 \mathrm{H})$, $2.39(\mathrm{~s}, 3 \mathrm{H}), 3.11(\mathrm{dd}, J=16.0,7.8 \mathrm{~Hz}, 1 \mathrm{H}), 3.52(\mathrm{dd}, J=16.0,9.4 \mathrm{~Hz}, 1 \mathrm{H}), 5.33(\mathrm{q}, J=7.8 \mathrm{~Hz}, 1 \mathrm{H})$, $5.66(\mathrm{ddq}, J=15.2,7.8,1.4 \mathrm{~Hz}, 1 \mathrm{H}), 5.93(\mathrm{dt}, J=15.2,6.3 \mathrm{~Hz}, 1 \mathrm{H}), 6.09(\mathrm{~s}, 1 \mathrm{H}), 6.74(\mathrm{~d}, J=8.4 \mathrm{~Hz}$, $1 \mathrm{H}) ; 7.41(\mathrm{~d}, J=8.4 \mathrm{~Hz}, 1 \mathrm{H}) ;{ }^{13} \mathrm{C} \mathrm{NMR}\left(\mathrm{CDCl}_{3}\right) \quad 13.3,19.2,21.4,33.1,86.4,106.7,111.3,113.9$, 114.2, 125.6, 127.3, 137.6, 151.0, 153.3, 161.4, 163.5; IR (neat) 1732, $1613 \mathrm{~cm}^{-1}$; HRMS m/z 256.1103 (calcd for $\mathrm{C}_{16} \mathrm{H}_{16} \mathrm{O}_{3}, 256.1099$ ).

\section{8,9-Dihydro-4-methyl-8-(2-methyl-1-propenyl)-2H-furo[2,3- $h]$-1-benzopyran-2-one}

Obtained in a 78\% yield: white solid, mp 140-146 ${ }^{\circ} \mathrm{C} ;{ }^{1} \mathrm{H}$ NMR $\left(\mathrm{CDCl}_{3}\right) \quad 1.81(\mathrm{~d}, J=1.2 \mathrm{~Hz}, 3 \mathrm{H})$, 
$1.82(\mathrm{~d}, J=1.4 \mathrm{~Hz}, 3 \mathrm{H}), 2.39(\mathrm{~d}, J=1.2 \mathrm{~Hz}, 3 \mathrm{H}), 3.04(\mathrm{dd}, J=16.0,8.8 \mathrm{~Hz}, 1 \mathrm{H}), 3.53(\mathrm{dd}, J=16.0$, $8.8 \mathrm{~Hz}, 1 \mathrm{H}), 5.45(\mathrm{dt}, J=8.8,1.2 \mathrm{~Hz}, 1 \mathrm{H}), 5.65(\mathrm{q}, J=8.8 \mathrm{~Hz}, 1 \mathrm{H}), 6.09(\mathrm{~d}, J=1.2 \mathrm{~Hz}, 1 \mathrm{H}), 6.73(\mathrm{~d}$, $J=8.4 \mathrm{~Hz}, 1 \mathrm{H}) ; 7.40(\mathrm{~d}, J=8.4 \mathrm{~Hz}, 1 \mathrm{H}) ;{ }^{13} \mathrm{C} \mathrm{NMR}\left(\mathrm{CDCl}_{3}\right) \quad 18.6,19.2,26.0,33.5,82.3,106.7$, 111.3, 114.0, 114.1, 123.9, 125.6, 139.6, 151.0, 153.3, 161.4, 163.6; IR (neat) 1727, $1610 \mathrm{~cm}^{-1}$; HRMS $m / z, 256.1103$ (calcd for $\mathrm{C}_{16} \mathrm{H}_{16} \mathrm{O}_{3}, 256.1099$ ).

8-(E-2-Butenyl)-8,9-dihydro-4-methyl-2H-furo[2,3- $\boldsymbol{h}]$-1-benzopyran-2-one (20). Obtained in an 83\% yield: white solid, mp 140-144 ${ }^{\circ} \mathrm{C} ;{ }^{1} \mathrm{H}$ NMR $\left(\mathrm{CDCl}_{3}\right) \quad 1.64(\mathrm{~s}, 3 \mathrm{H}), 1.68(\mathrm{~d}, J=6.8 \mathrm{~Hz}, 3 \mathrm{H}), 2.40$ (s, 3H), $3.18(\mathrm{dd}, J=16.4,8.0 \mathrm{~Hz}, 1 \mathrm{H}), 3.45(\mathrm{dd}, J=16.4,9.6 \mathrm{~Hz}, 1 \mathrm{H}), 5.33(\mathrm{t}, J=9.0 \mathrm{~Hz}, 1 \mathrm{H}), 5.68$ $(\mathrm{q}, J=6.8 \mathrm{~Hz}, 1 \mathrm{H}), 6.10(\mathrm{~s}, 1 \mathrm{H}), 6.76(\mathrm{~d}, J=8.4 \mathrm{~Hz}, 1 \mathrm{H}) ; 7.41(\mathrm{~d}, J=8.4 \mathrm{~Hz}, 1 \mathrm{H}) ;{ }^{13} \mathrm{C} \mathrm{NMR}\left(\mathrm{CDCl}_{3}\right)$ $10.8,13.4,19.2,31.2,90.5,106.5,111.3,114.1,124.0,125.6,133.8,151.0,153.3,161.4,164.0 ;$ IR (neat) $1728,1613 \mathrm{~cm}^{-1}$; HRMS m/z 256.11025 (calcd for $\mathrm{C}_{16} \mathrm{H}_{16} \mathrm{O}_{3}, 256.10994$ ).

\section{8,9-Dihydro-4,8-dimethyl-8-(2-methyl-1-propenyl)-2H-furo[2,3- $h$ ]-1-benzopyran-2-one}

(22).

Obtained in a $75 \%$ yield: white solid, mp $78-80{ }^{\circ} \mathrm{C} ;{ }^{1} \mathrm{H}$ NMR $\left(\mathrm{CDCl}_{3}\right) \quad 1.56(\mathrm{~s}, 3 \mathrm{H}), 1.72(\mathrm{~s}, 3 \mathrm{H}), 1.76$ (s, 3H), $2.39(\mathrm{~s}, 3 \mathrm{H}), 3.30(\mathrm{~d}, J=15.8 \mathrm{~Hz}, 1 \mathrm{H}), 3.40(\mathrm{~d}, J=15.8 \mathrm{~Hz}, 1 \mathrm{H}), 5.57(\mathrm{~s}, 1 \mathrm{H}), 6.09(\mathrm{~s}, 1 \mathrm{H})$, $6.74(\mathrm{~d}, J=8.5 \mathrm{~Hz}, 1 \mathrm{H}), 7.41(\mathrm{~d}, J=8.5 \mathrm{~Hz}, 1 \mathrm{H}) ;{ }^{13} \mathrm{C} \mathrm{NMR}\left(\mathrm{CDCl}_{3}\right) \quad 19.3,19.5,26.9,28.7,40.8$, $91.1,106.9,111.2,113.9,114.0,125.7,129.6,136.2,151.2,153.4,161.5,162.6$; IR (neat) 1728,1614 $\mathrm{cm}^{-1} ;$ HRMS $m / z, 270.1262$ (calcd for $\mathrm{C}_{17} \mathrm{H}_{18} \mathrm{O}_{3}, 270.1256$ ).

8,9-Dihydro-4,9-dimethyl-8-(E-1-propenyl)-2H-furo[2,3- $\boldsymbol{h}]-1$-benzopyran-2-one (3:2 mixture of trans- and cis-isomers 25a and $\mathbf{2 5} \mathbf{b}$ respectively). Obtained as a white solid in a $60 \%$ overall yield when a commercially available 3:2 mixture of trans,trans- and cis,cis-2,4-hexadienes was used. trans,trans-2,4-Hexadiene (95\% pure) gave a 20:1 ratio of $\mathbf{2 5 a}$ and $\mathbf{2 5 b}$ in a $70 \%$ yield. trans-Isomer (25a): ${ }^{1} \mathrm{H}$ NMR $\left(\mathrm{CDCl}_{3}\right) \quad 1.27(\mathrm{~d}, J=7.2 \mathrm{~Hz}, 3 \mathrm{H}), 1.82(\mathrm{dd}, J=7.0,1.5 \mathrm{~Hz}, 3 \mathrm{H}), 2.39(\mathrm{~s}, 3 \mathrm{H}), 3.74(\mathrm{p}$, $J=7.7 \mathrm{~Hz}, 1 \mathrm{H}), 5.52(\mathrm{t}, J=8.4 \mathrm{~Hz}, 1 \mathrm{H}), 5.73(\mathrm{ddq}, J=15.2,8.4,1.5 \mathrm{~Hz}, 1 \mathrm{H}), 5.88-6.01(\mathrm{~m}, 1 \mathrm{H})$, $6.10(\mathrm{~s}, 1 \mathrm{H}), 6.75(\mathrm{~d}, J=8.6 \mathrm{~Hz}, 1 \mathrm{H}) ; 7.40(\mathrm{~d}, J=8.6 \mathrm{~Hz}, 1 \mathrm{H}) ;{ }^{13} \mathrm{C} \mathrm{NMR}\left(\mathrm{CDCl}_{3}\right) \quad 15.1,18.3,19.3$, 38.3, 89.7, 107.0, 111.5, 114.4, 119.9, 125.6, 125.6, 132.7, 151.1, 153.4, 161.4, 162.7; IR (neat) 1720, 
$1605 \mathrm{~cm}^{-1}$. cis-Isomer (25b): ${ }^{1} \mathrm{H}$ NMR $\left(\mathrm{CDCl}_{3}\right) \quad 1.50(\mathrm{~d}, J=7.0 \mathrm{~Hz}, 3 \mathrm{H}), 1.77(\mathrm{dd}, J=6.6,1.5 \mathrm{~Hz}$, 3H), 2.39 (s, 3H), 3.49 (p, $J=7.2 \mathrm{~Hz}, 1 \mathrm{H}), 4.76(\mathrm{t}, J=7.3 \mathrm{~Hz}, 1 \mathrm{H}), 5.64(\mathrm{ddq}, J=15.4,7.7,1.5 \mathrm{~Hz}$, $1 \mathrm{H}), 5.82-5.91(\mathrm{~m}, 1 \mathrm{H}), 6.10(\mathrm{~s}, 1 \mathrm{H}), 6.74(\mathrm{~d}, J=8.6 \mathrm{~Hz}, 1 \mathrm{H}) ; 7.40(\mathrm{~d}, J=8.6 \mathrm{~Hz}, 1 \mathrm{H})$. The following ${ }^{13} \mathrm{C}$ NMR, IR and HRMS data were obtained on the mixture of stereoisomers: ${ }^{13} \mathrm{C}$ NMR $\left(\mathrm{CDCl}_{3}\right) \quad 15.1,18.0,18.1,18.2,19.3,38.0,38.3,41.5,89.7,93.7,106.9,107.0,111.4,111.5,114.3$, $114.4,118.3,119.9,125.6,125.7,125.8,129.0,131.0,132.7,151.1,151.4,153.3,153.4,161.2,161.3$, 162.7, 163.0; IR (neat) 1729, $1613 \mathrm{~cm}^{-1}$; HRMS m/z 256.1105 (calcd for $\mathrm{C}_{16} \mathrm{H}_{16} \mathrm{O}_{3}, 256.1099$ ).

\section{8,9-Dihydro-4-methyl-8-phenyl-8-(1-phenylethenyl)-2H-furo[2,3-h]-1-benzopyran-2-one (26).}

Obtained in a $10 \%$ yield: white solid, mp 60-64 ${ }^{\circ} \mathrm{C} ;{ }^{1} \mathrm{H}$ NMR $\left(\mathrm{CDCl}_{3}\right) \quad 2.38(\mathrm{~d}, J=1.2 \mathrm{~Hz}, 3 \mathrm{H}), 3.81$ $(\mathrm{d}, J=15.8 \mathrm{~Hz}, 1 \mathrm{H}), 3.83(\mathrm{~d}, J=15.8 \mathrm{~Hz}, 1 \mathrm{H}), 5.43(\mathrm{~d}, J=0.8 \mathrm{~Hz}, 1 \mathrm{H}), 5.60(\mathrm{~d}, J=0.8 \mathrm{~Hz}, 1 \mathrm{H}), 6.08$ $(\mathrm{d}, J=1.2 \mathrm{~Hz}, 1 \mathrm{H}), 6.86(\mathrm{~d}, J=7.6 \mathrm{~Hz}, 1 \mathrm{H}), 7.05-7.34(\mathrm{~m}, 10 \mathrm{H}), 7.31(\mathrm{~d}, J=7.6 \mathrm{~Hz}, 1 \mathrm{H}) ;{ }^{13} \mathrm{C} \mathrm{NMR}$ $\left(\mathrm{CDCl}_{3}\right) \quad 19.2,38.7,95.9,106.9,111.6,113.6,114.5,116.8,125.8,126.4,127.8,128.1,128.3,128.6$ 128.7, 139.4, 142.6, 150.9, 151.0, 153.3, 161.2, 162.3; IR (neat) 1727, $1612 \mathrm{~cm}^{-1}$; HRMS m/z 380.1419 (calcd for $\mathrm{C}_{26} \mathrm{H}_{20} \mathrm{O}_{3}, 380.1412$ ).

Dihydrofurocoumarin 28. Obtained in a $72 \%$ yield: white solid, mp $180-184{ }^{\circ} \mathrm{C} ;{ }^{1} \mathrm{H} \mathrm{NMR}\left(\mathrm{CDCl}_{3}\right)$ $1.00(\mathrm{~s}, 3 \mathrm{H}), 1.11(\mathrm{~s}, 3 \mathrm{H}), 1.66(\mathrm{~d}, J=13.8 \mathrm{~Hz}, 1 \mathrm{H}), 1.74(\mathrm{~s}, 3 \mathrm{H}), 1.81(\mathrm{~d}, J=17.6 \mathrm{~Hz}, 1 \mathrm{H}), 1.90(\mathrm{~d}, J$ $=17.6 \mathrm{~Hz}, 1 \mathrm{H}), 1.99(\mathrm{~d}, J=13.8 \mathrm{~Hz}, 1 \mathrm{H}), 2.25(\mathrm{~s}, 3 \mathrm{H}), 2.37(\mathrm{~d}, J=1.2 \mathrm{~Hz}, 3 \mathrm{H}), 3.12(\mathrm{~d}, J=16.0 \mathrm{~Hz}$,

$1 \mathrm{H}), 3.14(\mathrm{~d}, J=16.0 \mathrm{~Hz}, 1 \mathrm{H}), 5.51(\mathrm{~s}, 1 \mathrm{H}), 6.07(\mathrm{~d}, J=1.2 \mathrm{~Hz}, 1 \mathrm{H}), 7.20(\mathrm{~s}, 1 \mathrm{H}) ;{ }^{13} \mathrm{C} \mathrm{NMR}\left(\mathrm{CDCl}_{3}\right)$ $8.8,19.3,24.2,28.6,30.2,31.0,43.4,44.4,48.1,89.5,107.9,110.7,113.4,117.4,122.9,123.7,138.6$, 153.3, 153.4, 160.9, 162.2; IR (neat) 1720, $1616 \mathrm{~cm}^{-1}$; HRMS m/z 324.1731 (calcd for $\mathrm{C}_{21} \mathrm{H}_{24} \mathrm{O}_{3}$, 324.1725).

\section{2,3-Dihydro-5,9-dimethyl-2-(E-2-phenylethenyl)-7H-furo[3,2-g][1]-benzopyran-7-one}

Obtained in a $73 \%$ yield: white solid, mp 140-144 ${ }^{\circ} \mathrm{C} ;{ }^{1} \mathrm{H}$ NMR $\left(\mathrm{CDCl}_{3}\right) 2.32(\mathrm{~s}, 3 \mathrm{H}), 2.39(\mathrm{~d}, J=1.0$ $\mathrm{Hz}, 3 \mathrm{H}), 3.16(\mathrm{dd}, J=15.6,7.7 \mathrm{~Hz}, 1 \mathrm{H}), 3.52(\mathrm{dd}, J=15.6,9.2 \mathrm{~Hz}, 1 \mathrm{H}), 5.49(\mathrm{dt}, J=9.2,7.7 \mathrm{~Hz}, 1 \mathrm{H})$, $6.11(\mathrm{q}, J=1.0 \mathrm{~Hz}, 1 \mathrm{H}), 6.36(\mathrm{dd}, J=15.8,7.7 \mathrm{~Hz}, 1 \mathrm{H}), 6.73(\mathrm{~d}, J=15.8 \mathrm{~Hz}, 1 \mathrm{H}), 7.27-7.48(\mathrm{~m}, 6 \mathrm{H})$; 
${ }^{13} \mathrm{C} \mathrm{NMR}\left(\mathrm{CDCl}_{3}\right) \quad 8.8,19.3,36.2,85.2,108.2,111.2,113.9,117.5,119.2,123.1,126.8,127.8$, 128.5, 129.0, 132.3, 133.2, 136.1, 153.2, 153.4, 161.4, 170.0; IR (neat) 1714, 1616, $1585 \mathrm{~cm}^{-1}$; HRMS $m / z 318.1263$ (calcd for $\mathrm{C}_{21} \mathrm{H}_{18} \mathrm{O}_{3}, 318.1256$ ).

2-(E-1-Butenyl)-2,3-dihydro-5,9-dimethyl-7H-furo[3,2-g][1]-benzopyran-7-one (30). Obtained in an $75 \%$ yield: white solid, mp $127-129{ }^{\circ} \mathrm{C} ;{ }^{1} \mathrm{H}$ NMR $\left(\mathrm{CDCl}_{3}\right) \quad 1.04(\mathrm{t}, J=7.6 \mathrm{~Hz}, 3 \mathrm{H}), 2.19$ (m, 2H), $2.29(\mathrm{~s}, 3 \mathrm{H}), 2.38(\mathrm{~d}, J=1.2 \mathrm{~Hz}, 3 \mathrm{H}), 3.04(\mathrm{ddd}, J=15.6,8.1,1.2 \mathrm{~Hz}, 1 \mathrm{H}), 3.40(\mathrm{ddd}, J=15.6$, 9.0, 1.0 Hz, 1H), $5.27(\mathrm{q}, J=8.2 \mathrm{~Hz}, 1 \mathrm{H}), 5.65(\mathrm{ddt}, J=15.3,7.8,1.7 \mathrm{~Hz}, 1 \mathrm{H}), 5.92(\mathrm{dtd}, J=15.3,6.2$, $0.8 \mathrm{~Hz}, 1 \mathrm{H}), 6.09(\mathrm{~d}, J=1.2 \mathrm{~Hz}, 1 \mathrm{H}), 7.22(\mathrm{~s}, 1 \mathrm{H}) ;{ }^{13} \mathrm{C} \mathrm{NMR}\left(\mathrm{CDCl}_{3}\right) \quad$ 8.8, 13.3, 19.3, 25.4, 36.0, 85.6, 108.0, 111.0, 113.7, 117.4, 123.5, 127.7, 137.2, 153.2, 153.3, 161.5, 162.1; IR (neat) 1713,1614 $\mathrm{cm}^{-1} ;$ HRMS m/z, 270.1262 (calcd for $\mathrm{C}_{17} \mathrm{H}_{18} \mathrm{O}_{3}, 270.1256$ ).

\section{2,3-Dihydro-5,9-dimethyl-2-(2-methyl-1-propenyl)-7H-furo[3,2-g][1]benzopyran-7-one}

(31).

Obtained in a $79 \%$ yield: white solid, mp 176-180 ${ }^{\circ} \mathrm{C} ;{ }^{1} \mathrm{H} \mathrm{NMR}\left(\mathrm{CDCl}_{3}\right) \quad 1.81(\mathrm{~d}, J=1.2 \mathrm{~Hz}, 3 \mathrm{H})$, $2.28(\mathrm{~s}, 3 \mathrm{H}), 2.38(\mathrm{~d}, J=1.0 \mathrm{~Hz}, 3 \mathrm{H}), 2.98(\mathrm{ddd}, J=15.5,8.6,1.3 \mathrm{~Hz}, 1 \mathrm{H}), 3.39(\mathrm{ddd}, J=15.5,8.7$, $1.0 \mathrm{~Hz}, 1 \mathrm{H}), 5.44(\mathrm{dt}, J=9.1,1.1 \mathrm{~Hz}, 1 \mathrm{H}), 5.57(\mathrm{q}, J=8.6 \mathrm{~Hz}, 1 \mathrm{H}), 6.09(\mathrm{~d}, J=1.2 \mathrm{~Hz}, 1 \mathrm{H}), 7.22(\mathrm{~s}$, $1 \mathrm{H}) ;{ }^{13} \mathrm{C} \mathrm{NMR}\left(\mathrm{CDCl}_{3}\right) \quad 8.6,18.4,19.1,25.9,36.2,81.4,107.7,110.7,113.4,117.0,123.5,124.0$, 138.7, 152.9, 153.1, 161.4, 161.8; IR (neat) 1704, $1622 \mathrm{~cm}^{-1}$; HRMS m/z 270.1262 (calcd for $\mathrm{C}_{17} \mathrm{H}_{18} \mathrm{O}_{3}$, 270.1256).

2-(E-2-Butenyl)-2,3-dihydro-5,9-dimethyl-7H-furo[3,2-g][1]-benzopyran-7-one (33). Obtained in a 75\% yield: white solid, mp 180-186 ${ }^{\circ} \mathrm{C} ;{ }^{1} \mathrm{H}$ NMR $\left(\mathrm{CDCl}_{3}\right) \quad 1.82(\mathrm{~d}, J=1.2 \mathrm{~Hz}, 3 \mathrm{H}), 2.28(\mathrm{~s}, 3 \mathrm{H})$, $2.38(\mathrm{~d}, J=1.2 \mathrm{~Hz}, 3 \mathrm{H}), 2.97(\mathrm{ddd}, J=15.6,7.5,0.9 \mathrm{~Hz}, 1 \mathrm{H}), 3.39(\mathrm{ddd}, J=15.6,8.8,0.8 \mathrm{~Hz}, 1 \mathrm{H})$, $5.44(\mathrm{dt}, J=9.2,1.2 \mathrm{~Hz}, 1 \mathrm{H}), 5.57(\mathrm{q}, J=8.8 \mathrm{~Hz}, 1 \mathrm{H}), 6.09(\mathrm{~d}, J=1.2 \mathrm{~Hz}, 1 \mathrm{H}), 7.22(\mathrm{~s}, 1 \mathrm{H}) ;{ }^{13} \mathrm{C}$ $\operatorname{NMR}\left(\mathrm{CDCl}_{3}\right) \quad 8.8,18.7,19.3,26.1,36.4,81.7,108.0,111.0,113.6,117.3,123.8,124.3,139.0$, 153.2, 153.4, 161.6, 162.1; IR (neat) 1698, $1613 \mathrm{~cm}^{-1}$; HRMS m/z 270.1262 (calcd for $\mathrm{C}_{17} \mathrm{H}_{18} \mathrm{O}_{3}$, 270.1256). 
2,3-Dihydro-3,5,9-trimethyl-8-(E-1-propenyl)-7H-furo[3,2-g][1]-benzopyran-7-one (3:2 mixture

of trans- and cis-isomers $\mathbf{3 4 a}$ and $\mathbf{3 4 b}$ respectively). Obtained as a white solid in a $60 \%$ overall yield when a commercially available 3:2 mixture of trans,trans- and cis,cis-2,4-hexadienes was used. trans,trans-2,4-Hexadiene (95\% pure) gave a 20:1 ratio of $\mathbf{3 4 a}$ and $\mathbf{3 4 b}$ in a $70 \%$ yield. trans-Isomer (34a): ${ }^{1} \mathrm{H} \mathrm{NMR}\left(\mathrm{CDCl}_{3}\right) \quad 1.20(\mathrm{~d}, J=7.2 \mathrm{~Hz}, 3 \mathrm{H}), 1.80(\mathrm{dd}, J=6.5,1.5 \mathrm{~Hz}, 3 \mathrm{H}), 2.29(\mathrm{~s}, 3 \mathrm{H}), 2.40$ (s, $3 \mathrm{H}), 3.54(\mathrm{p}, J=7.4 \mathrm{~Hz}, 1 \mathrm{H}), 5.21(\mathrm{t}, J=8.5 \mathrm{~Hz}, 1 \mathrm{H}), 5.57-5.75(\mathrm{~m}, 1 \mathrm{H}), 5.80-6.00(\mathrm{~s}, 1 \mathrm{H}), 6.10(\mathrm{~s}$, 1H), $7.17(\mathrm{~s}, 1 \mathrm{H}) . \quad$ cis-Isomer (34b): ${ }^{1} \mathrm{H}$ NMR $\left(\mathrm{CDCl}_{3}\right) \quad 1.36(\mathrm{~d}, J=6.9 \mathrm{~Hz}, 3 \mathrm{H}), 1.80(\mathrm{dd}, J=6.5,1.5$ $\mathrm{Hz}, 3 \mathrm{H}), 2.28(\mathrm{~s}, 3 \mathrm{H}), 2.40(\mathrm{~s}, 3 \mathrm{H}), 3.25(\mathrm{p}, J=7.2 \mathrm{~Hz}, 1 \mathrm{H}), 4.65(\mathrm{t}, J=8.3 \mathrm{~Hz}, 1 \mathrm{H}), 5.57-5.75(\mathrm{~m}$, 1H), 5.80-6.00 (m, 1H), $6.10(\mathrm{~s}, 1 \mathrm{H}), 7.14(\mathrm{~s}, 1 \mathrm{H})$. The following ${ }^{13} \mathrm{C}$ NMR, IR and HRMS data were obtained on the mixture of stereoisomers: ${ }^{13} \mathrm{C}$ NMR $\left(\mathrm{CDCl}_{3}\right) \quad 8.8,16.5,17.7,18.3,19.4,39.9,42.6$, 89.0, 93.5, 108.1, 111.0, 111.1, 113.9, 116.2, 116.7, 126.2, 129.29, 129.7, 131.6, 131.9, 153.3, 153.4, 160.8, 162.0; IR (neat) 1714, $1620 \mathrm{~cm}^{-1}$; HRMS m/z, 270.1262 (calcd for $\mathrm{C}_{17} \mathrm{H}_{18} \mathrm{O}_{3}, 270.1256$ ).

\section{Derivatization of the Dihydrofurocoumarins}

Preparation of dihydrofurocoumarin 39. A modified solvomercuration procedure was used. ${ }^{5}$ Coumarin 23 (0.25 mmol, $64 \mathrm{mg})$ and $\mathrm{Hg}(\mathrm{OAc})_{2}(0.5 \mathrm{mmol}, 163 \mathrm{mg})$ were dissolved in $2 \mathrm{~mL}$ of a $1: 1$ ether/water solution and 2 drops of $48 \% \mathrm{HClO}_{4}$ was added to dissolve the precipitated mercury(II) oxide. The reaction mixture was stirred at room temperature for $24 \mathrm{~h}$, then quenched with a solution of $\mathrm{NaBH}_{4}(0.3 \mathrm{mmol}, 11.4 \mathrm{mg})$ in $5 \%$ aq $\mathrm{NaOH}(0.5 \mathrm{~mL})$. The resulting reaction mixture was stirred for $10 \mathrm{~min}$, gently acidified with $10 \mathrm{~mL}$ of satd $\mathrm{NH}_{4} \mathrm{Cl}$ (use of $\mathrm{HCl}$ results in only a $10 \%$ yield of the product 39), and extracted with diethyl ether. Then the ether extract was dried over anhydrous $\mathrm{MgSO}_{4}$ and concentrated. The resulting residue was purified by column chromatography using silica gel as a solid phase and 4:1 hexanes/ethyl acetate as the eluent to afford the final product after solvent removal.

8,9-Dihydro-8-(1-hydroxy-1-methylethyl)-4,8-dimethyl-2H-furo[2,3- $h$ ]-1-benzopyran-2-one (39). Obtained in a $65 \%$ yield: white solid, mp $118-120{ }^{\circ} \mathrm{C} ;{ }^{1} \mathrm{H}$ NMR $\left(\mathrm{CDCl}_{3}\right) \quad 1.26(\mathrm{~s}, 3 \mathrm{H}), 1.39$ (s, 3H), 
$1.47(\mathrm{~s}, 3 \mathrm{H}), 2.39$ (d, $J=1.2 \mathrm{~Hz}, 3 \mathrm{H}), 2.98(\mathrm{~d}, J=16.0 \mathrm{~Hz}, 1 \mathrm{H}), 3.59(\mathrm{~d}, J=16.0,1 \mathrm{H}), 6.11(\mathrm{~d}, J=$

$1.2 \mathrm{~Hz}, 1 \mathrm{H}), 6.75(\mathrm{~d}, J=8.4 \mathrm{~Hz}, 1 \mathrm{H}), 7.41(\mathrm{~d}, J=8.4 \mathrm{~Hz}, 1 \mathrm{H}) ;{ }^{13} \mathrm{C} \mathrm{NMR}\left(\mathrm{CDCl}_{3}\right) \quad$ 19.3, 23.0, 24.5, 24.9, 34.9, 74.5, 97.1, 106.8, 111.4, 114.2, 114.3, 125.6, 151.3, 153.3, 161.4, 162.8; IR (neat) 3469, 1727, $1614 \mathrm{~cm}^{-1}$; HRMS m/z 274.1212 (calcd for $\mathrm{C}_{16} \mathrm{H}_{18} \mathrm{O}_{4}, 274.1205$ ).

Preparation of the diol 40. A modified dihydroxylation procedure was used. ${ }^{6}$ A solution of coumarin $23(0.25 \mathrm{mmol}, 64 \mathrm{mg})$ and NMO dihydrate $(0.55 \mathrm{mmol}, 61 \mathrm{mg})$ in 8:1 acetone-water $(2$ $\mathrm{mL})$ was treated with $1 \mathrm{~mL}$ of $5 \% \mathrm{OsO}_{4}$ solution in ethanol $(0.02 \mathrm{mmol}, 5 \mathrm{mg})$. The reaction was stirred at $70{ }^{\circ} \mathrm{C}$ for $2 \mathrm{~h}$, then diluted with $10 \%$ aq $\mathrm{NaHCO}_{3}(2 \mathrm{~mL})$ and extracted with ethyl acetate. The organic extract was washed with water and aq $\mathrm{NH}_{4} \mathrm{Cl}$ solution, dried over anhydrous $\mathrm{MgSO}_{4}$ for 4 $\mathrm{h}$ and concentrated. The resulting residue was purified by column chromatography using silica gel as a solid phase and 4:1 hexanes/ethyl acetate as the eluent to afford the final product after solvent removal.

\section{8,9-Dihydro-4,8-dimethyl-8-(1,2-dihydroxy-1-methylethyl)-2H-furo[2,3- $h]$-1-benzopyran-2-one}

(40). Obtained as a pair of diastereomers in a $96 \%$ yield: white solid, mp $163-173{ }^{\circ} \mathrm{C}$; ${ }^{1} \mathrm{H}$ NMR $\left(\mathrm{CDCl}_{3}\right) \quad 1.26(\mathrm{~s}, 3 \mathrm{H}), 1.37(\mathrm{~s}, 3 \mathrm{H}), 1.47(\mathrm{~s}, 6 \mathrm{H}), 2.39(\mathrm{~d}, J=0.8 \mathrm{~Hz}, 6 \mathrm{H}), 2.70-4.40(\mathrm{~m}, 8 \mathrm{H}), 6.11(\mathrm{q}$, $J=0.8 \mathrm{~Hz}, 1 \mathrm{H}), 6.70-6.77(\mathrm{~m}, 2 \mathrm{H}), 7.41(\mathrm{~d}, J=8.4 \mathrm{~Hz}, 2 \mathrm{H}) ;{ }^{13} \mathrm{C} \mathrm{NMR}\left(\mathrm{CDCl}_{3}\right) \quad 19.3,20.2,20.5$, $23.1,23.3,35.0,35.2,66.4,66.9,75.3,76.2,95.9,97.4,106.8,106.9,111.5,111.7,113.8,114.0$, $114.4,14.5,125.6,125.7,151.2,151.3,153.2,153.3,161.2,161.3,162.2,162.3$; IR (neat) 3406, 1709, $1608 \mathrm{~cm}^{-1}$; HRMS m/z 290.1160 (calcd for $\mathrm{C}_{16} \mathrm{H}_{18} \mathrm{O}_{5}, 290.1154$ ).

Preparation of the coumarin 41. A standard dehydrogenation procedure was used. ${ }^{7}$ A solution of coumarin $9(0.25 \mathrm{mmol}, 64 \mathrm{mg})$ and DDQ $(0.50 \mathrm{mmol}, 60 \mathrm{mg})$ in $1 \mathrm{~mL}$ of toluene was stirred at 100 ${ }^{\circ} \mathrm{C}$ for $24 \mathrm{~h}$. Then the resulting reaction mixture was cooled to room temperature, filtered and the filtrate was concentrated. The resulting residue was purified by column chromatography using silica gel as a stationary phase and 8:1 hexanes/ethyl acetate as the eluent to afford the final product after solvent removal. 
4-Methylbenzo[b]-2H-furo[2,3- $\boldsymbol{h}]$-1-benzopyran-2-one (41). Obtained in a 90\% yield: white solid, mp 216-219 ${ }^{\circ} \mathrm{C} ;{ }^{1} \mathrm{H}$ NMR $\left(\mathrm{CDCl}_{3}\right) \quad 2.53(\mathrm{~d}, J=1.2 \mathrm{~Hz}, 3 \mathrm{H}), 6.07(\mathrm{~d}, J=1.2 \mathrm{~Hz}, 1 \mathrm{H}), 7.45(\mathrm{td}, J=$ 7.3, $1.0 \mathrm{~Hz}, 1 \mathrm{H}), 7.49(\mathrm{~d}, J=8.6 \mathrm{~Hz}, 1 \mathrm{H}), 7.53(\mathrm{td}, J=7.6,1.2 \mathrm{~Hz}, 1 \mathrm{H}), 7.60(\mathrm{dt}, J=7.3,1.0 \mathrm{~Hz}, 1 \mathrm{H})$,

$7.67(\mathrm{~d}, J=8.6 \mathrm{~Hz}, 1 \mathrm{H}), 8.42(\mathrm{ddd}, J=7.6,1.2,0.8 \mathrm{~Hz}, 1 \mathrm{H}) ;{ }^{13} \mathrm{C} \mathrm{NMR}\left(\mathrm{CDCl}_{3}\right) \quad 19.6,108.5,111.7$, $111.7,113.2,115.2,122.1,123.4,123.8,124.0,128.0,149.7,153.5,156.3,158.5,160.8$; IR (neat) cm ${ }^{1}$; HRMS m/z 250.0635 (calcd for $\mathrm{C}_{16} \mathrm{H}_{14} \mathrm{O}_{3}, 250.0630$ ).

\section{References}

(1) Charlton, J. L.; Chee, G.; McColeman, H. Can J. Chem. 1995, 73, 1454.

(2) Harayama, T.; Katsuno, K.; Nishioka, H.; Fujii, M.; Nishita, Y.; Ishii, H.; Kaneko, Y. Heterocycles 1994, 39, 613.

(3) Teizo, S.; Koichi, T. Chem. Lett. 2001, 110.

(4) Rozhkov, R. V.; Larock, R. C. Org. Lett. 2003, 5, 797.

(5) Noland, W. E. Org. Syn. Coll. Vol. 6; John Wiley and Sons, NY 1988, 766.

(6) Panek, J. S.; Cirillo, P. F. J. Am. Chem. Soc. 1990, 112, 4873.

(7) Via, L. D.; Gia, O.; Magno, S. M.; Santana, L.; Teijeira, M.; Uriarte, E. J. Med. Chem. 1999, 42, 4405. 


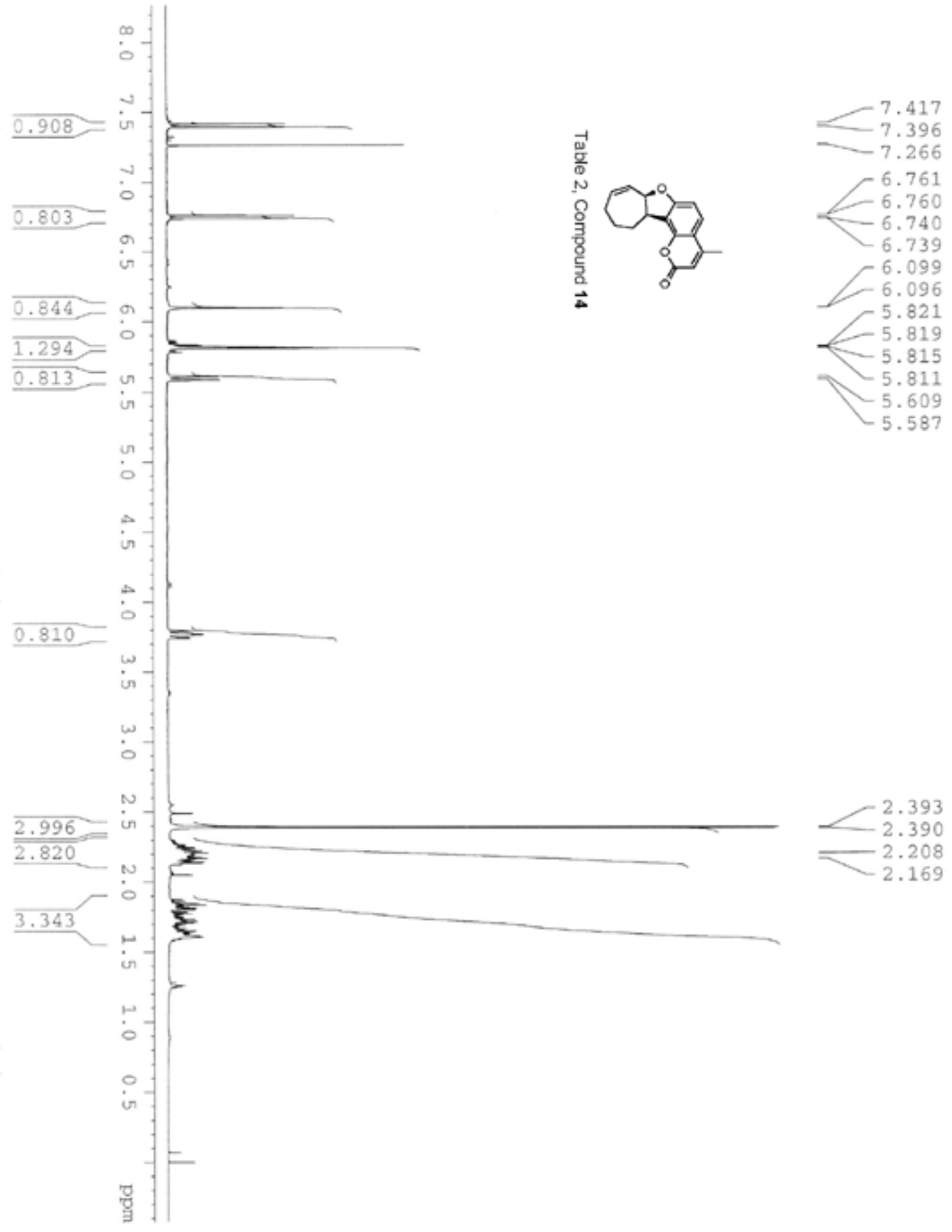



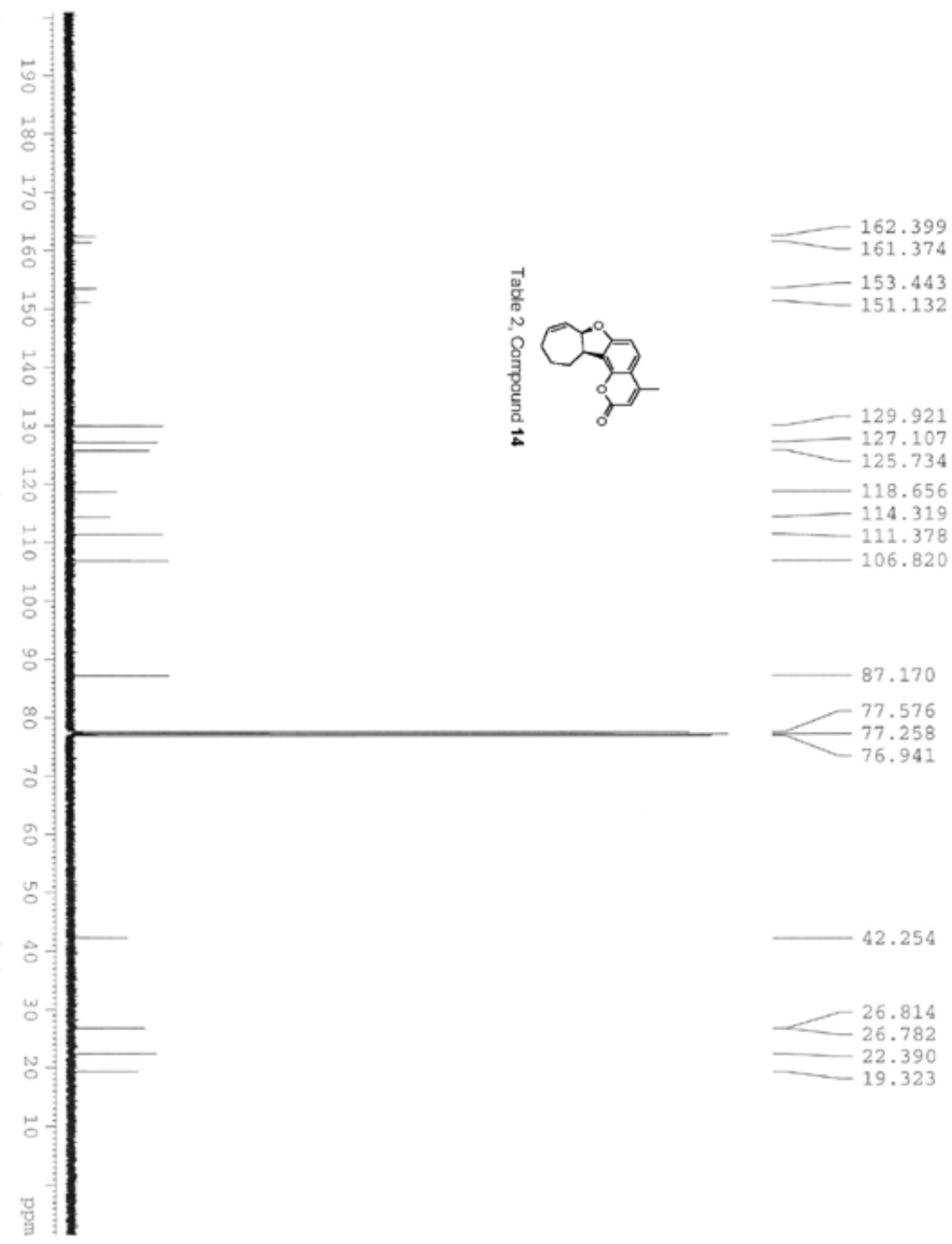

42.254

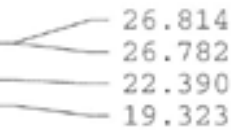



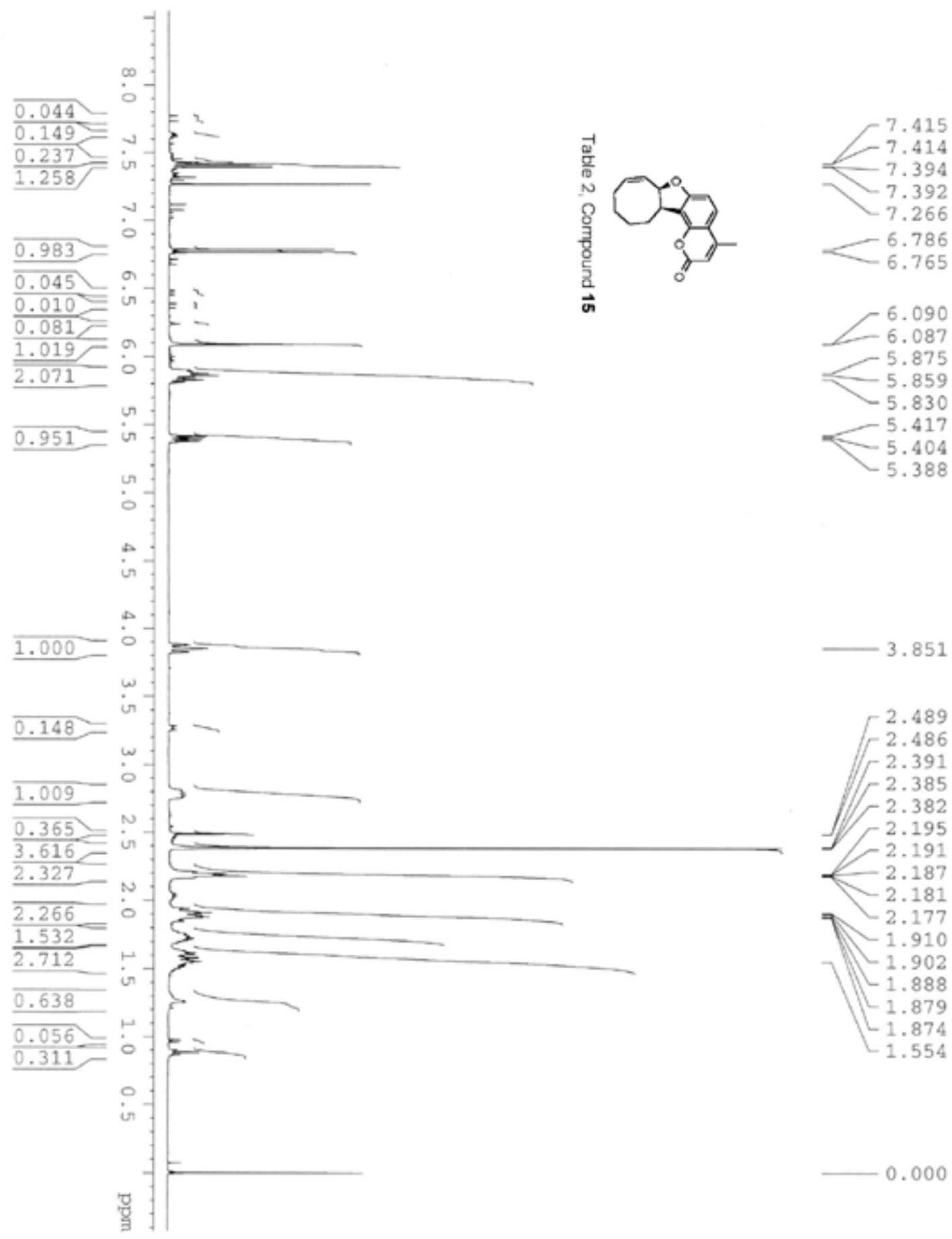

$-3.851$

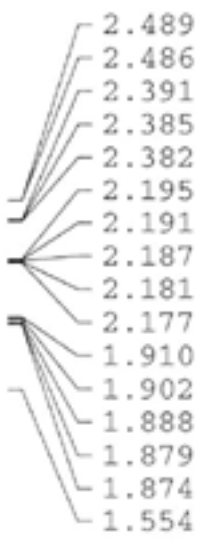

0.000 

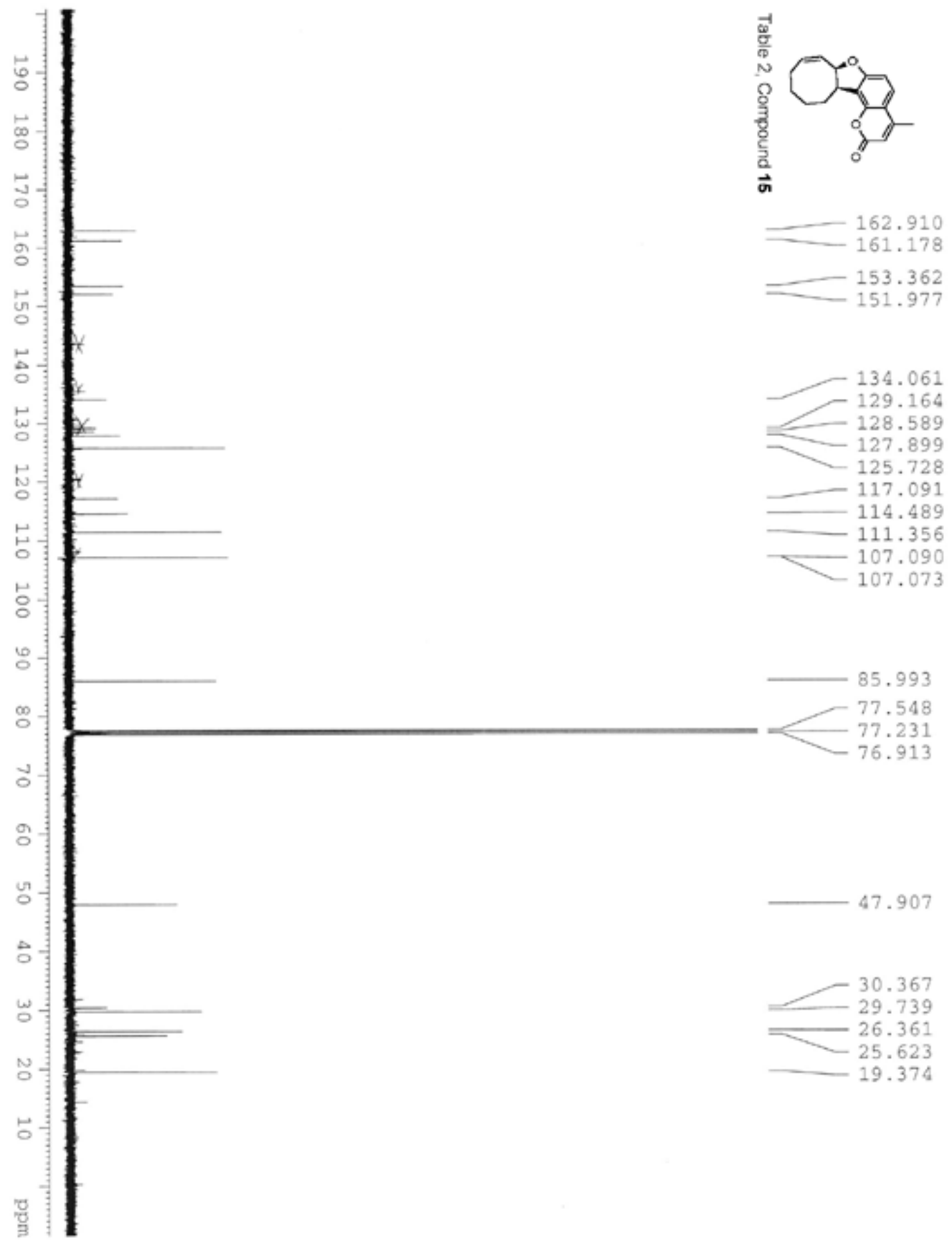

77.548 77.231

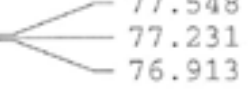

47.907

30.367 29.739 26.361

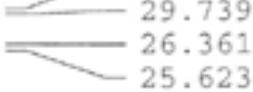
19.374 

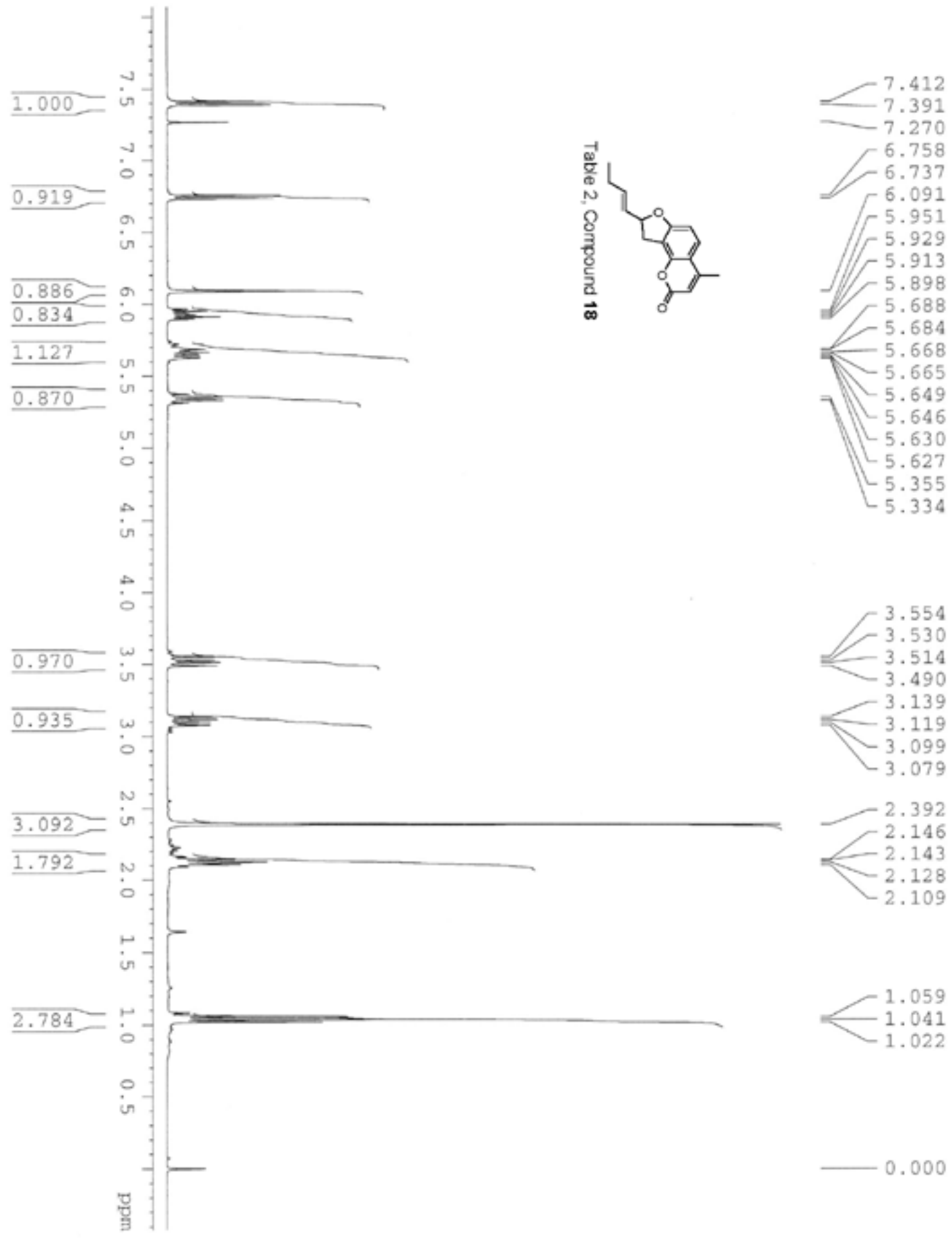

$-2.128$

$\leftarrow 2.109$

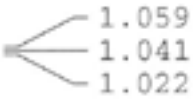

0.000 

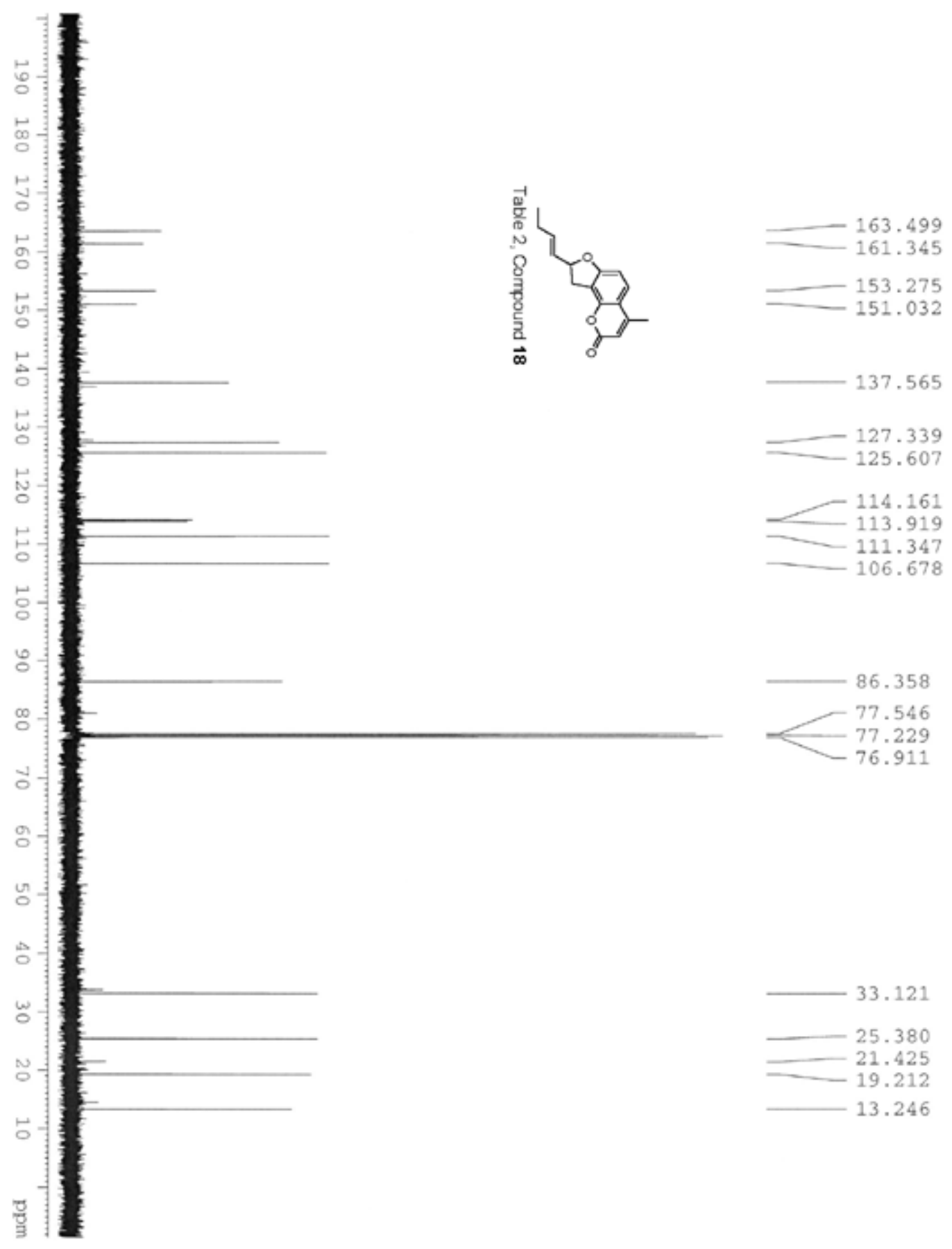

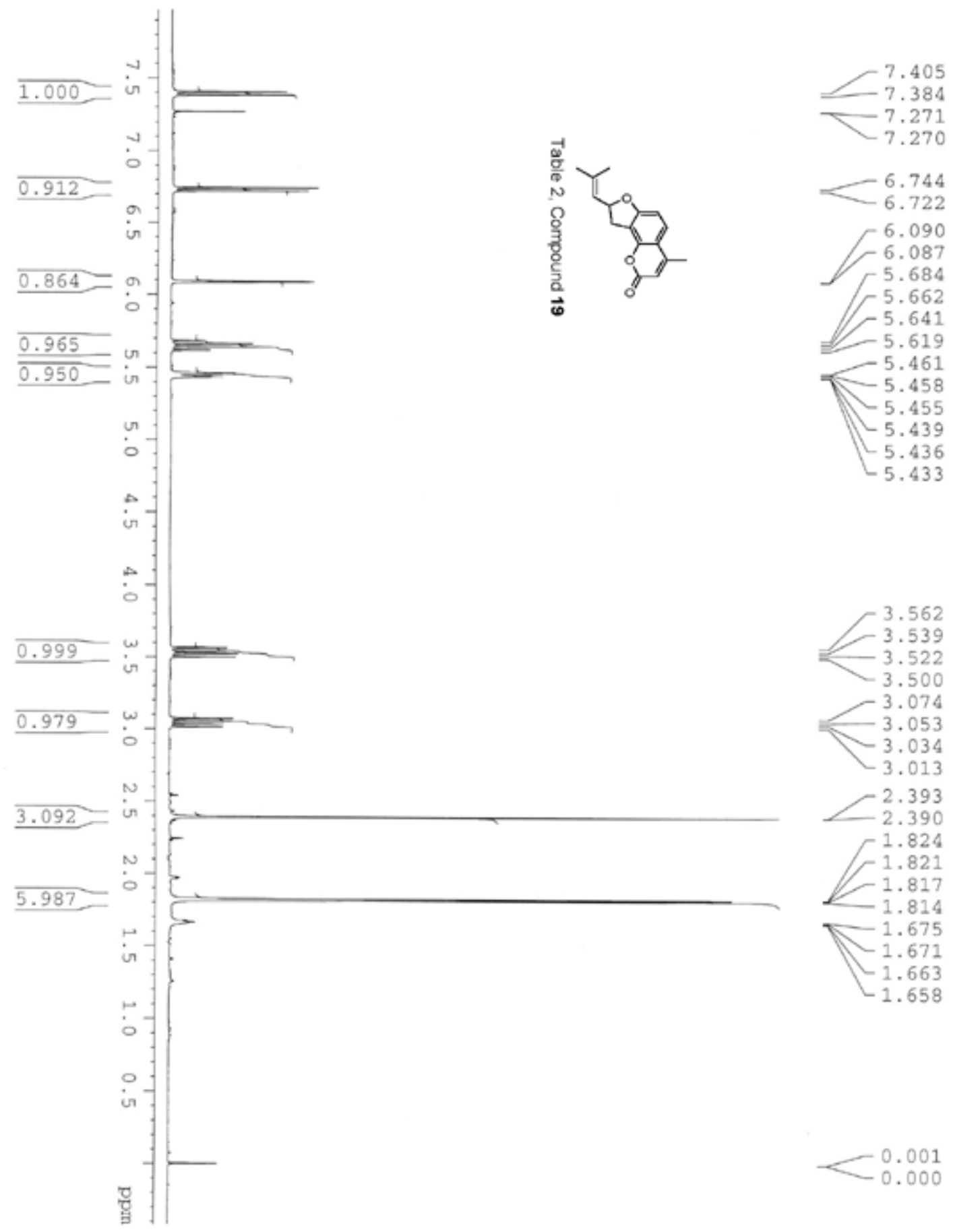

3.074

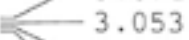

$-3.034$

$-3.013$

$-2.393$

2.390

- 1.824

1.821

$-1.817$

1.614
-1.675

$-1.671$

1.663

$-1.658$

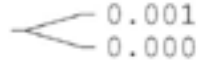




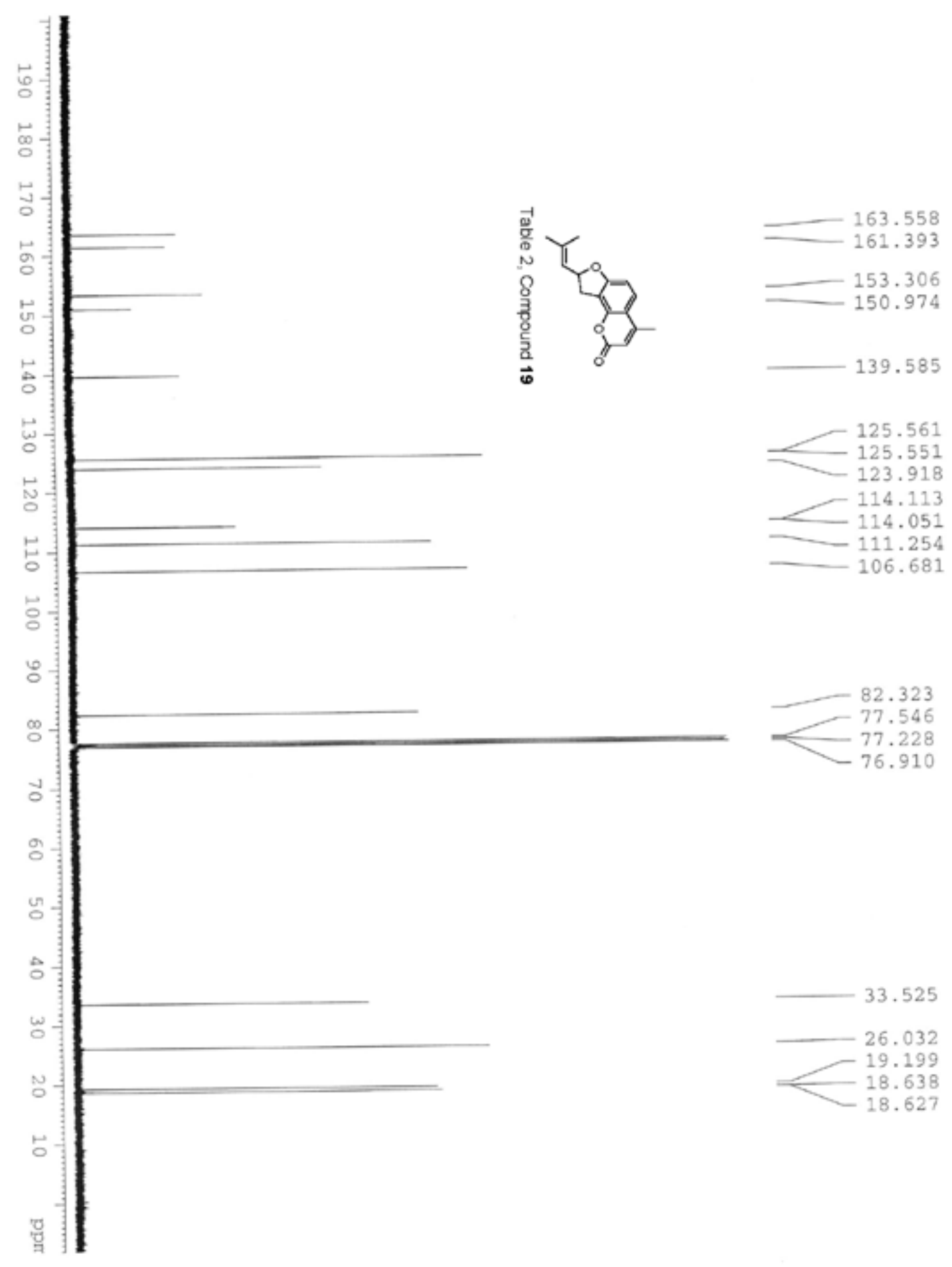



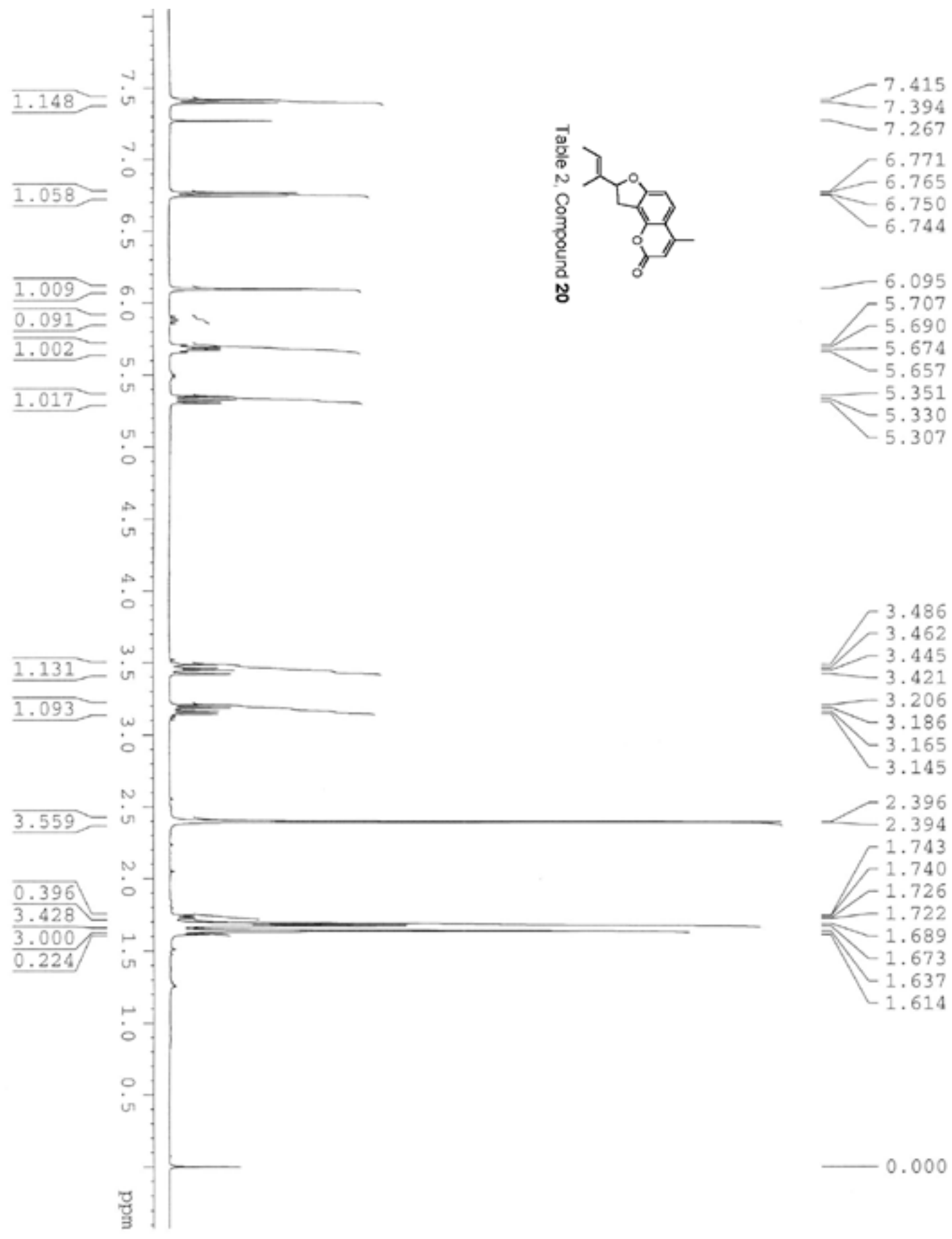

3.186
-3.165

$-3.165$

3.145

$-2.396$

2.396
-2.793

$\Gamma^{1.743}$

$-1.740$

$-1.726$

1.722
-1.689
-1.673

$-1.673$

$-1.637$

1.614

0.000 


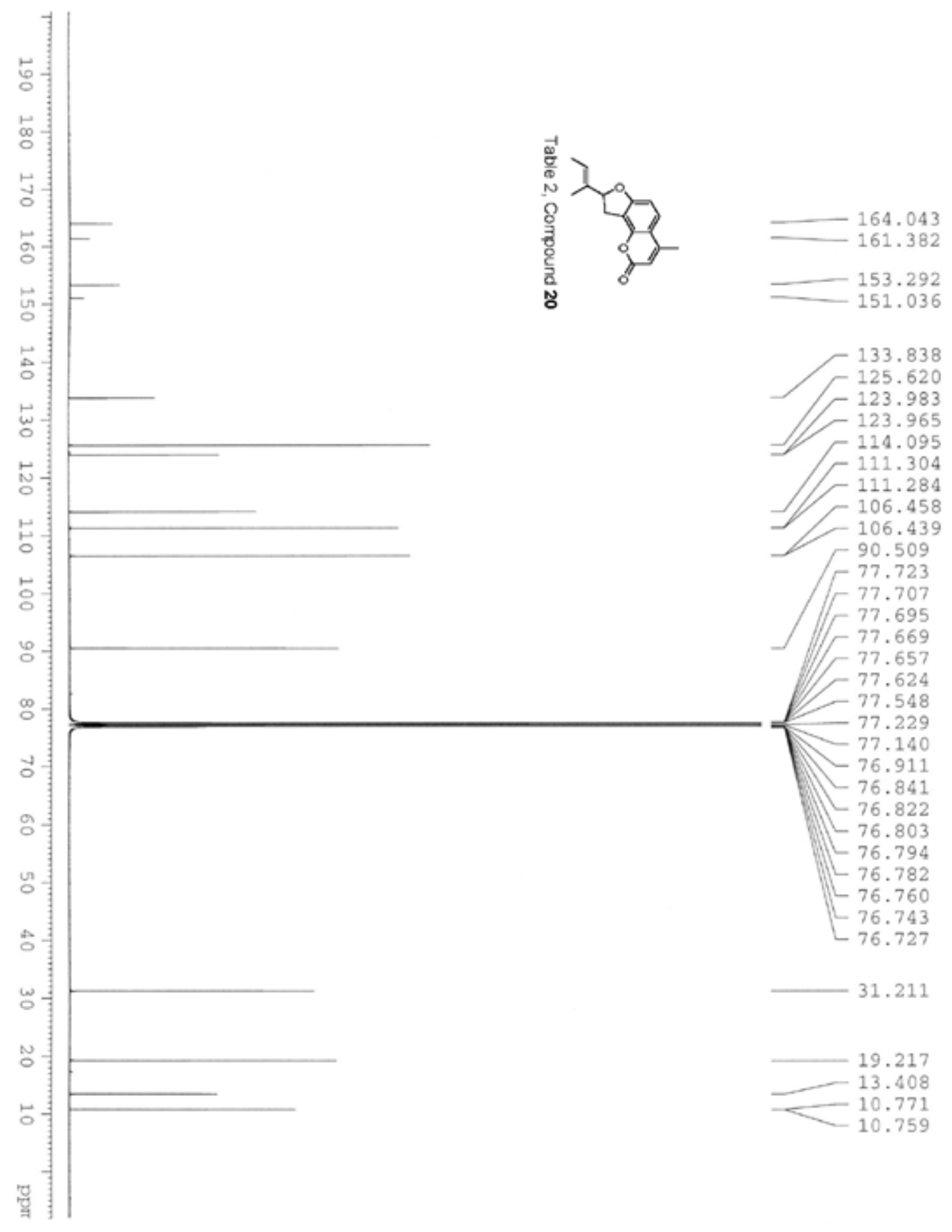




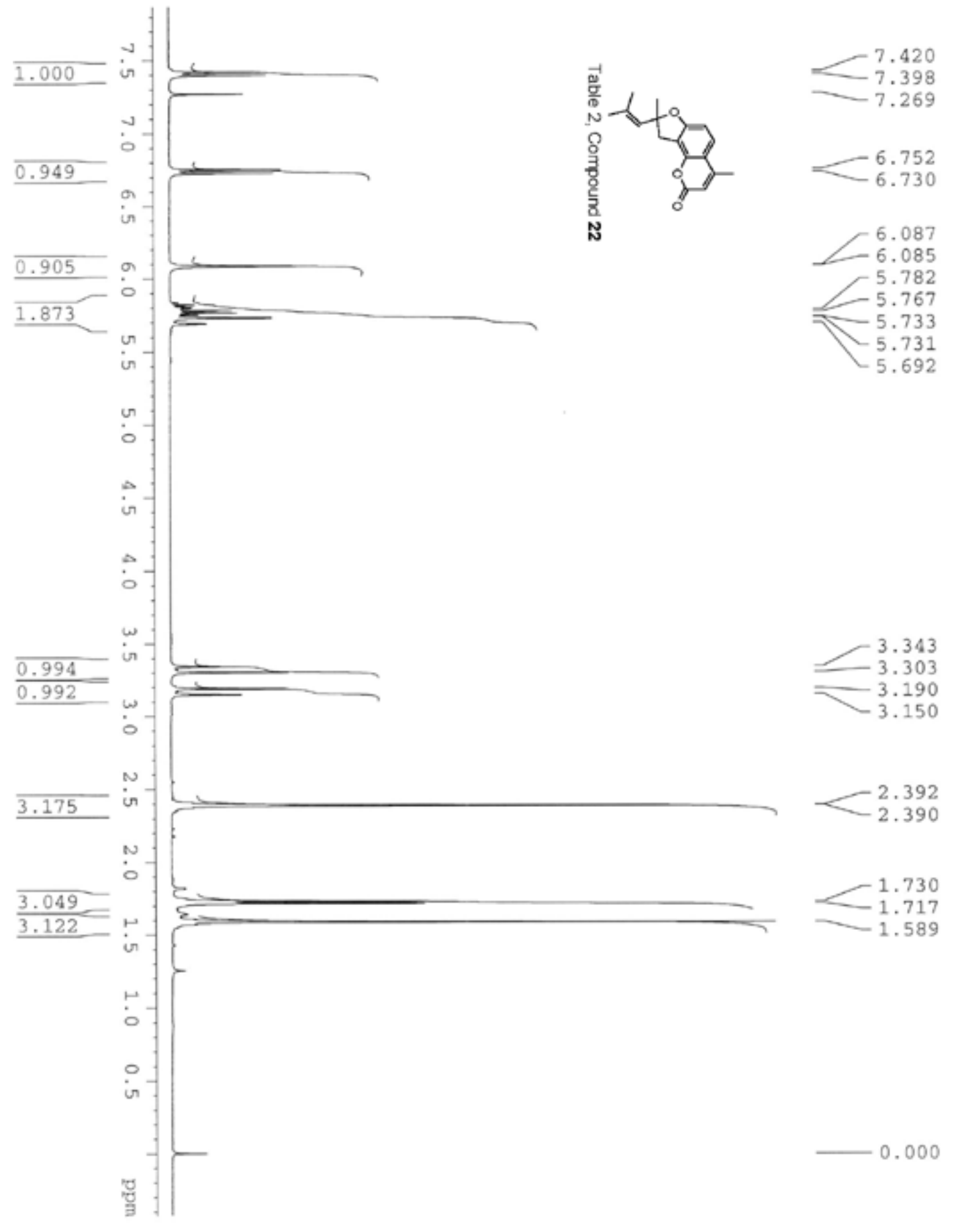




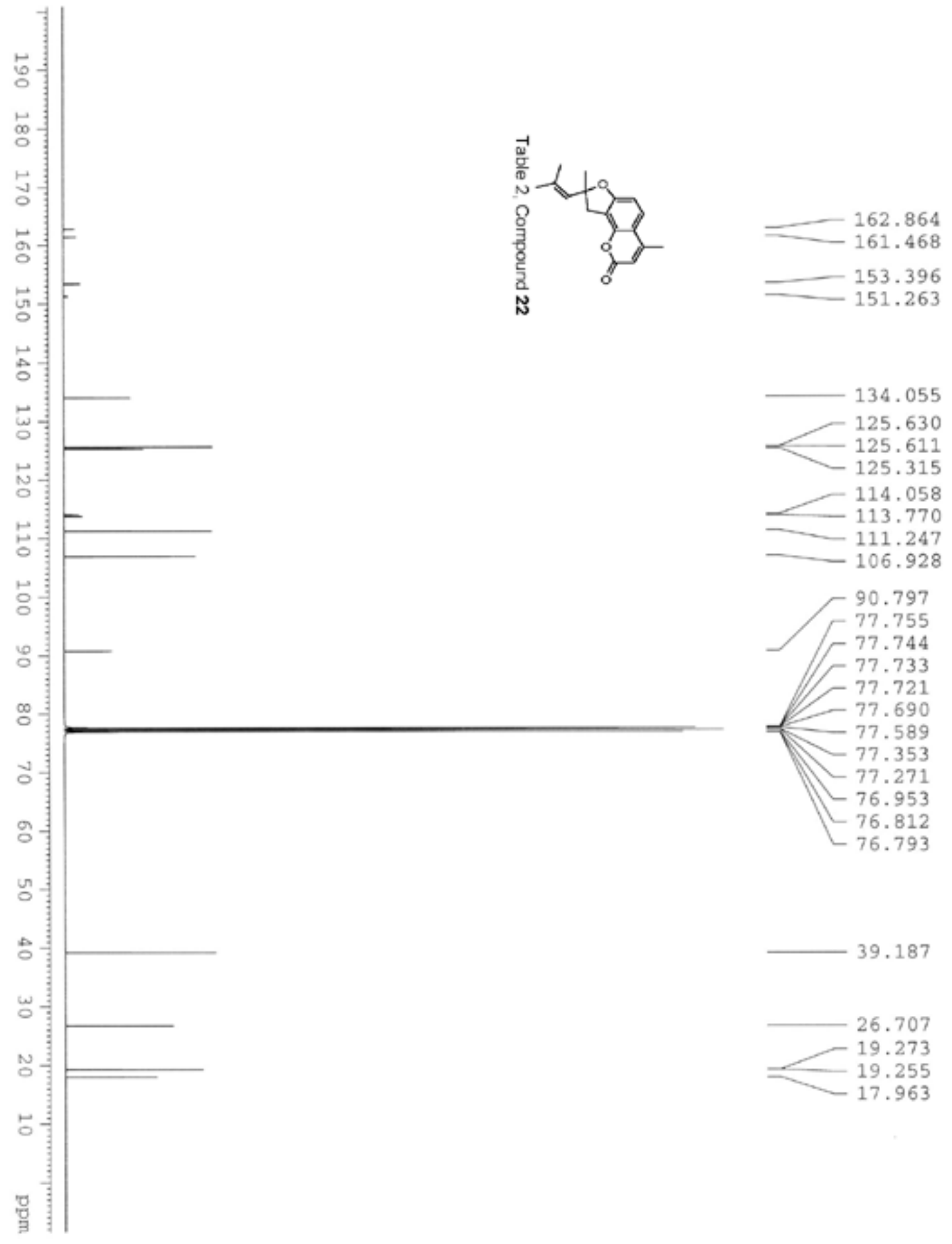



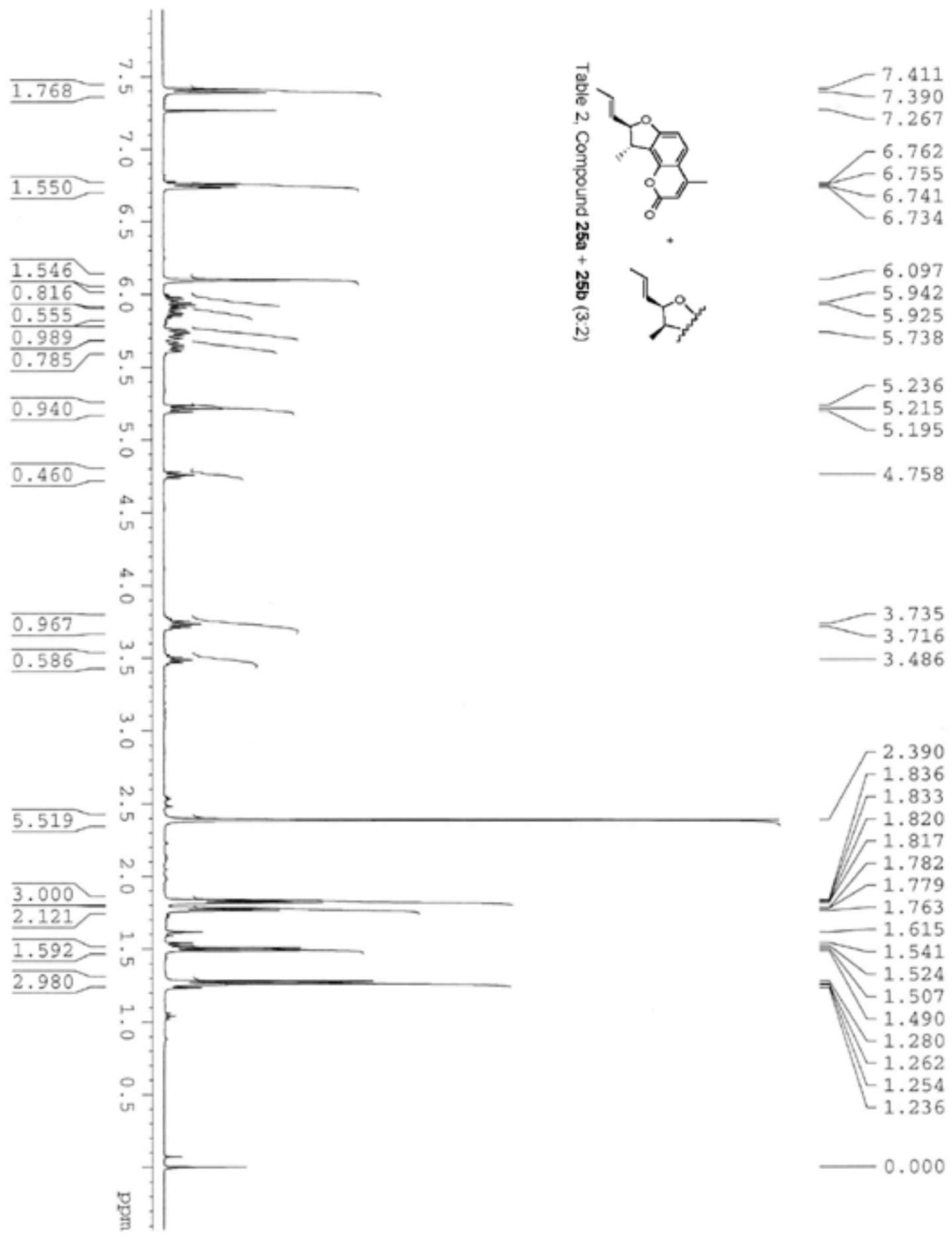

-3.735
-3.716

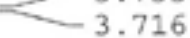
3.486

$\Gamma^{2.390} 1.836$

$r^{1.833}$

$-1.820$

- 1.817

1.782
-1.779

1.763

1.615

e 1.541

$=1.524$

$\simeq-1.507$

$-1.490$

a -1.280

- 1.262

$-1.254$

ᄂ 1.236

0.000 


$$
\text { 1? }
$$



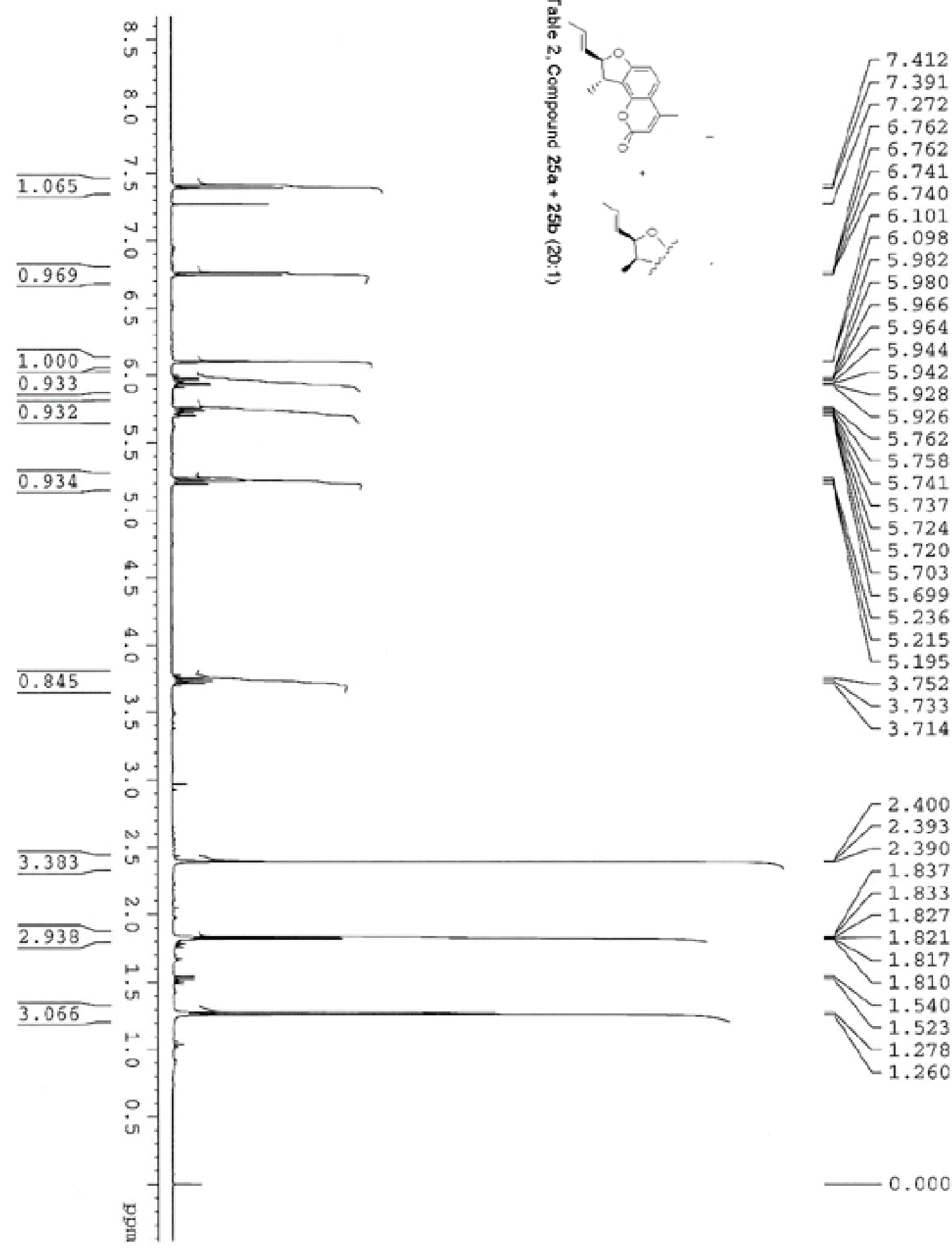

- 1.837

$-1.833$

$-1.827$

1. 821

$\checkmark 1.817$

$\checkmark 1.810$

$\checkmark 1.540$

1.278
-1.260

$-1.260$

$-0.000$ 

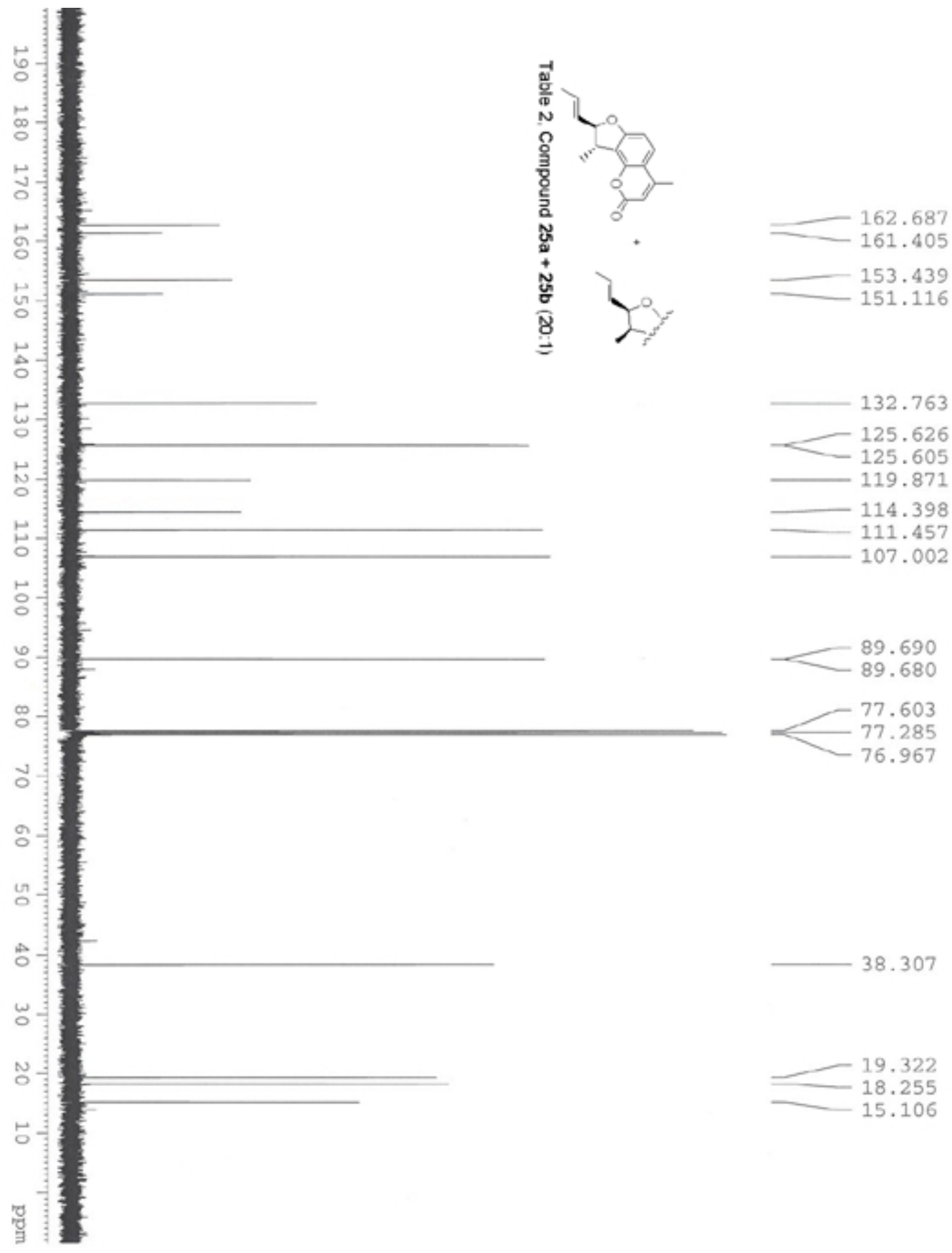

38.307

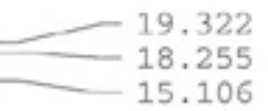




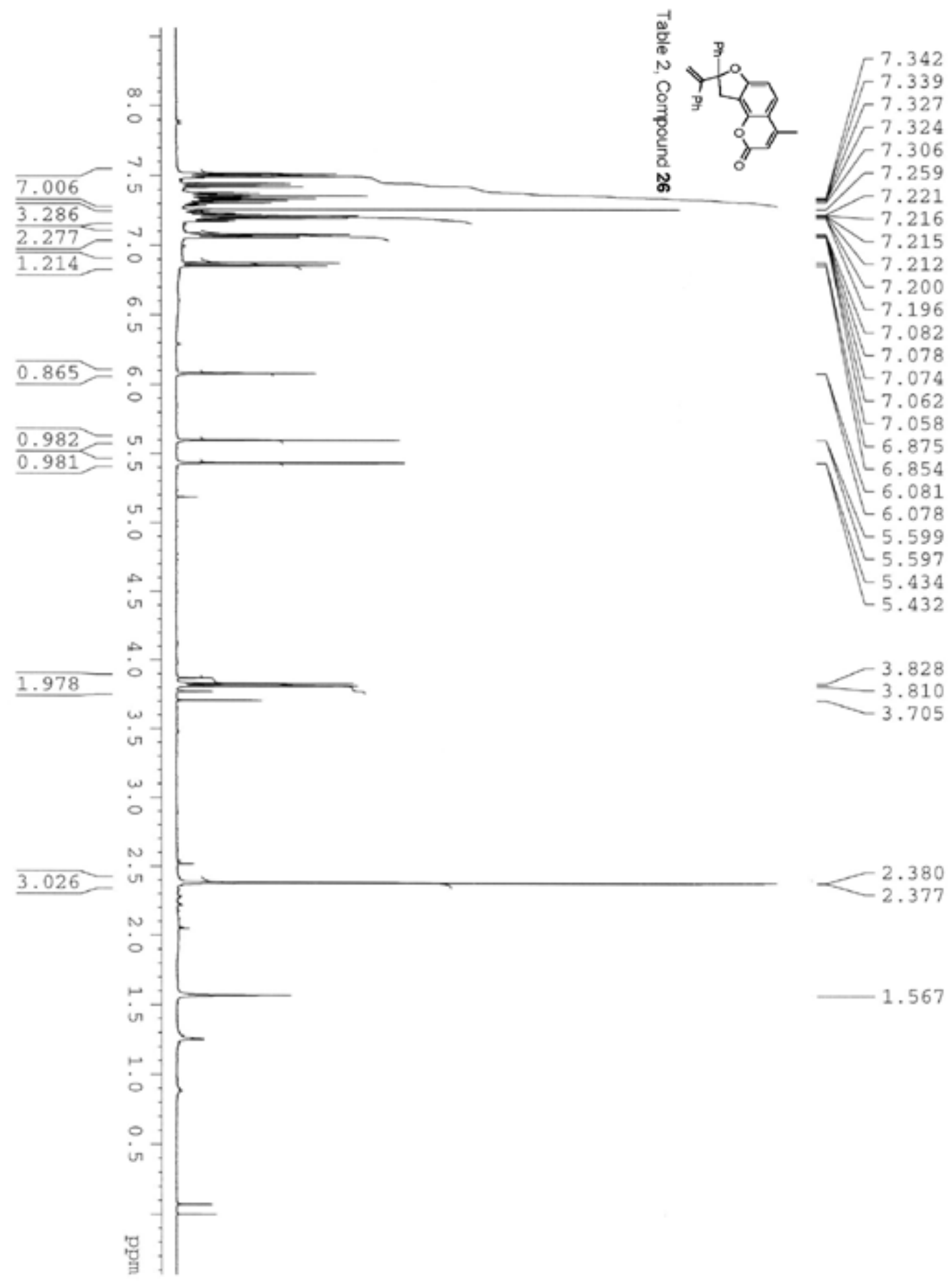



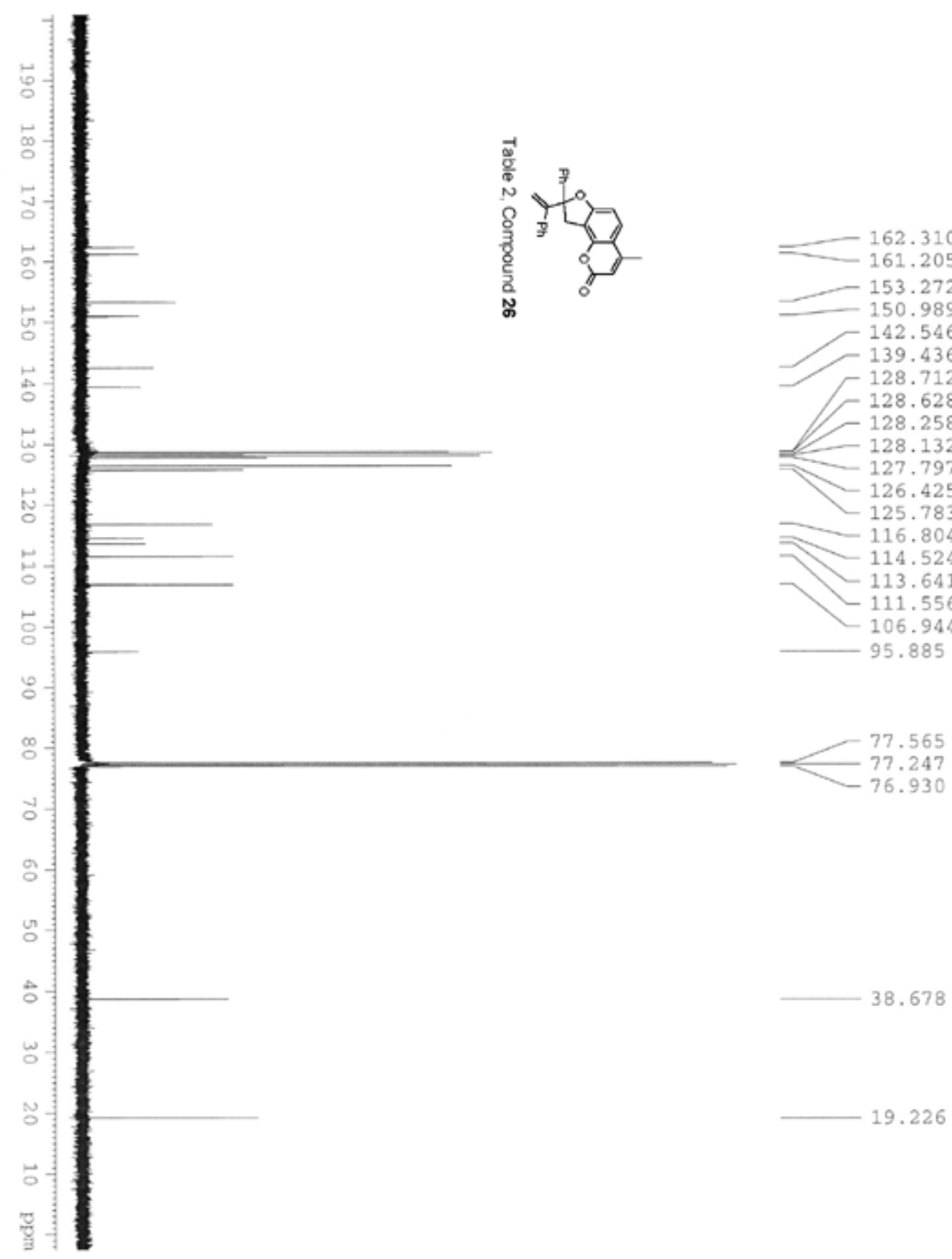


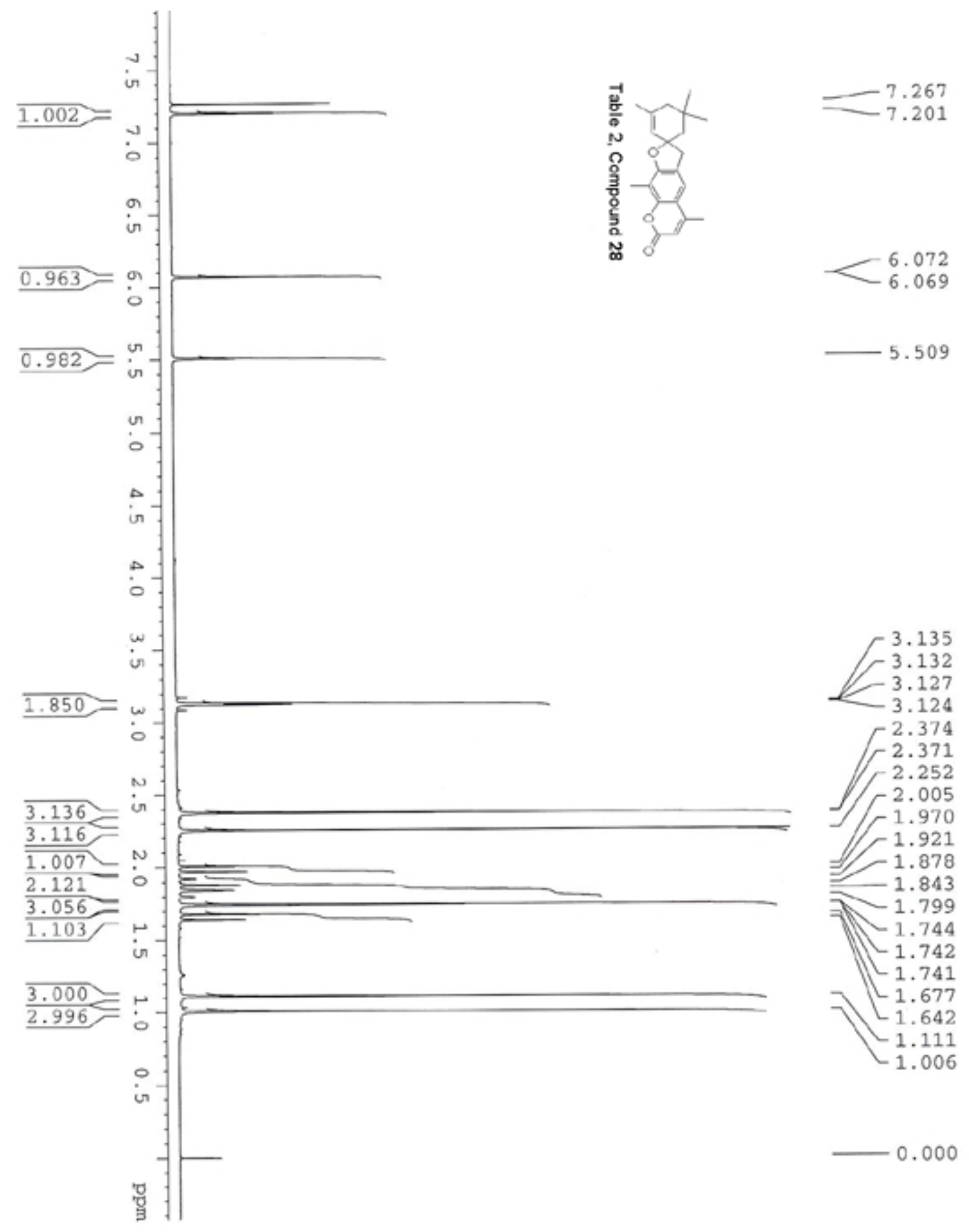



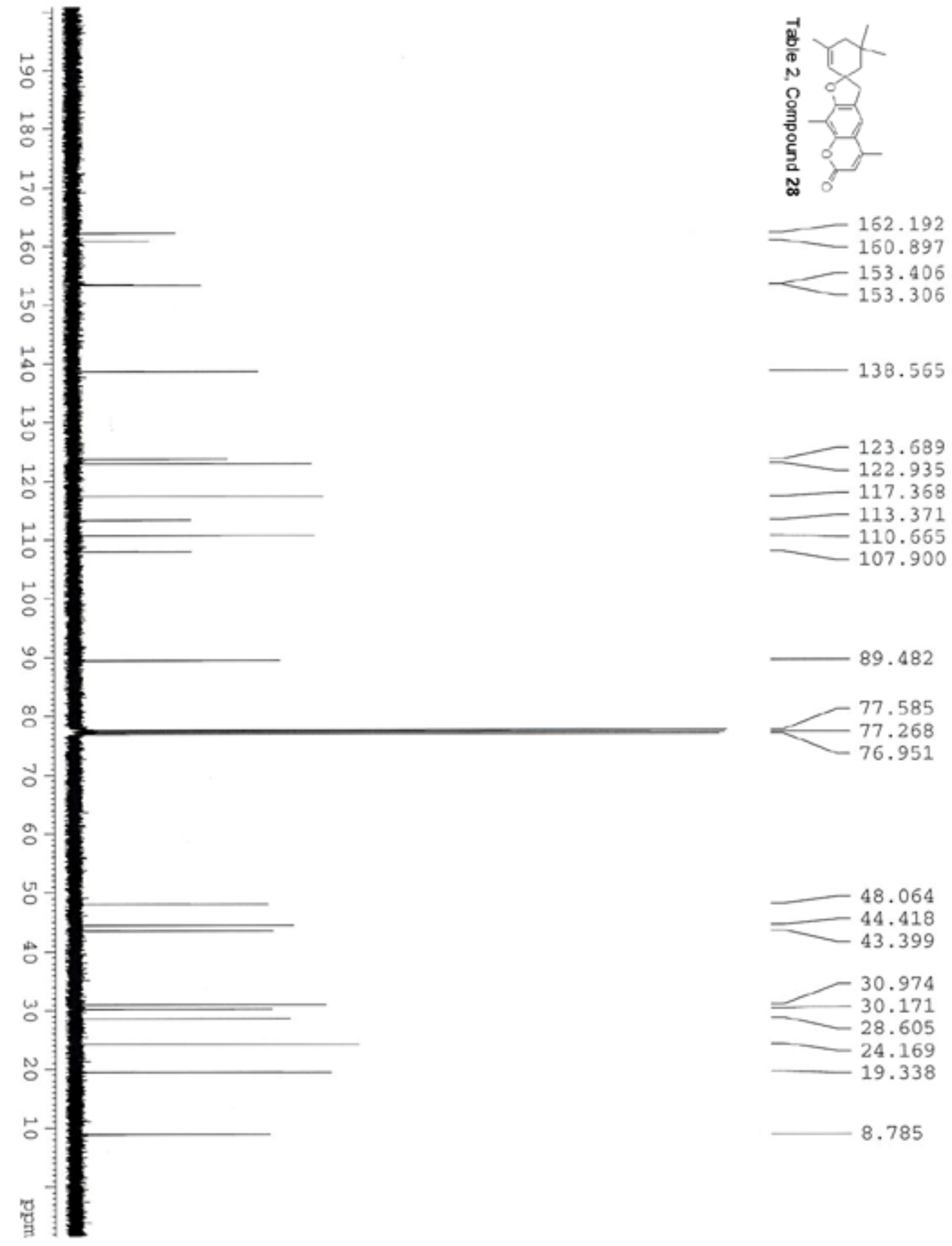


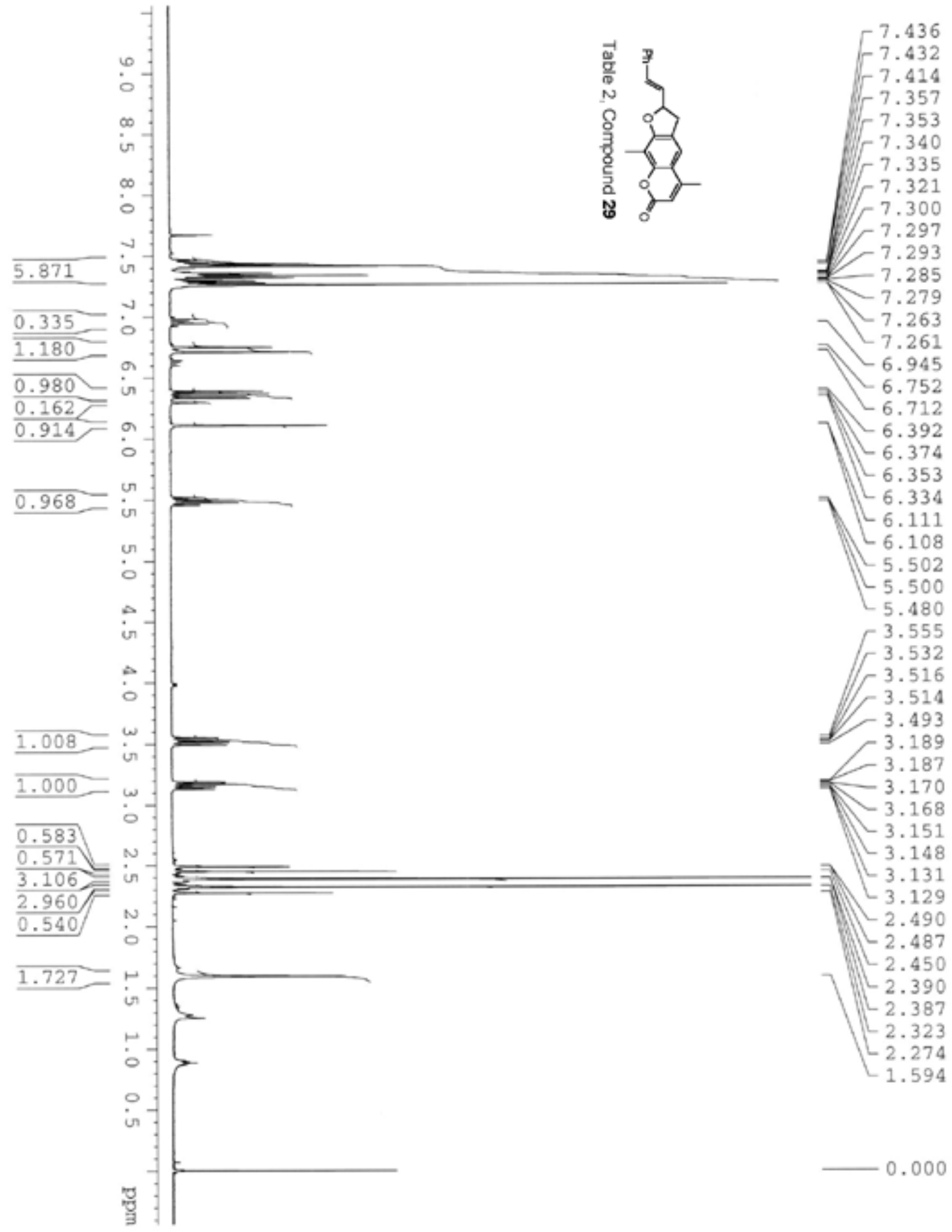



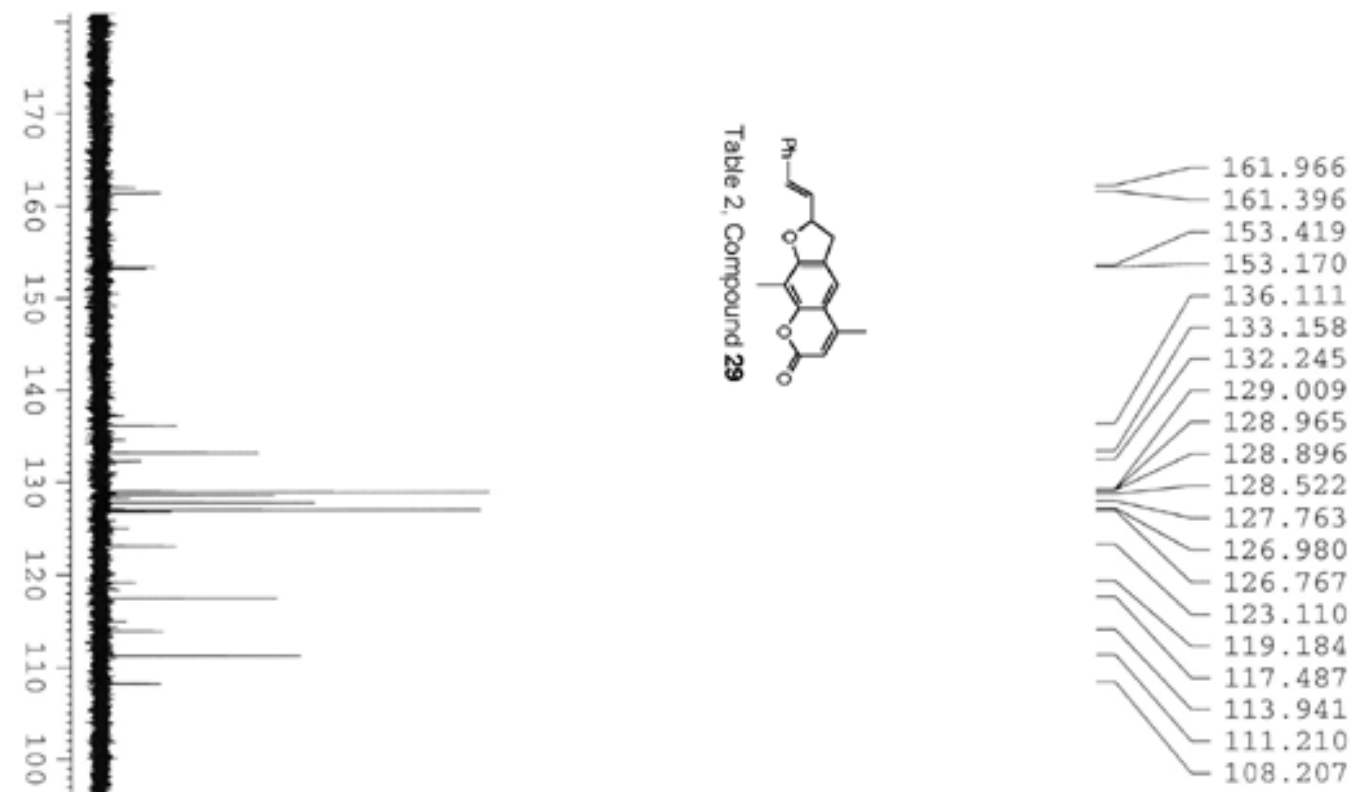

$\circ$

电

0

8

0

$\Delta$

w

N

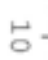

$\circ-$

"ृ

36.164

19.340

8.836 


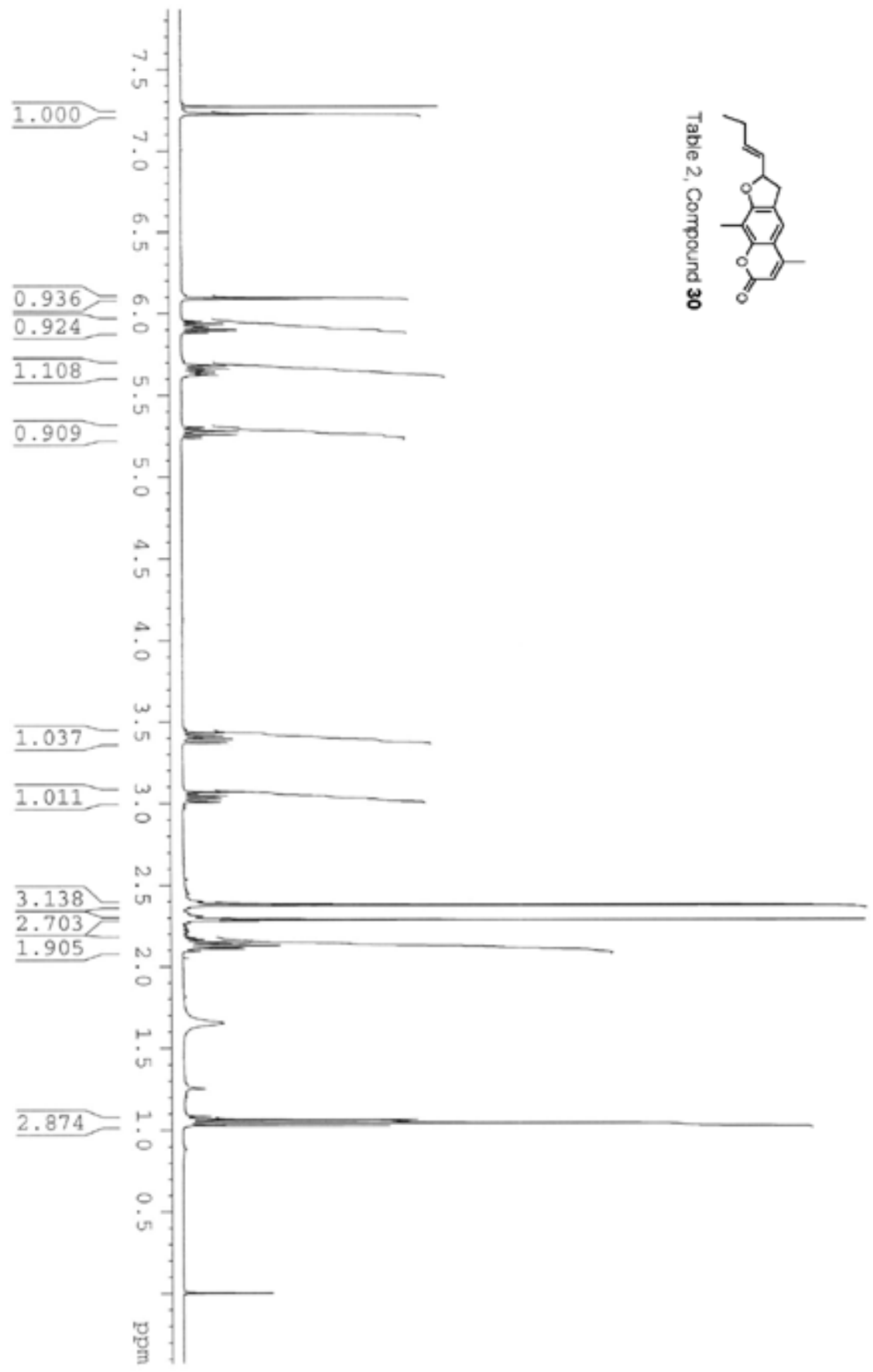

$-7.267$

$-7.223$

6.092

$-6.089$

$-5.938$

$-5.936$

$-5.900$

$-5.898$

$-5.682$

$-5.662$

$-5.624$

$-5.281$

5.261

- 3.434

3.412

$-3.395$

$-3.392$

$\sqrt{-3.373}$

3.370
-3.068

$-3.065$

$-3.048$

-3.045
-3.029

- 3.009

$-2.379$

2.379
-2.376
-2.289

$\longrightarrow 2.281$

$\sim 2.148$

$-2.144$

$7-2.129$

$-2.114$

2.110
1.653

$-1.653$

1.062
-1.044

$-1.025$

$-0.000$ 


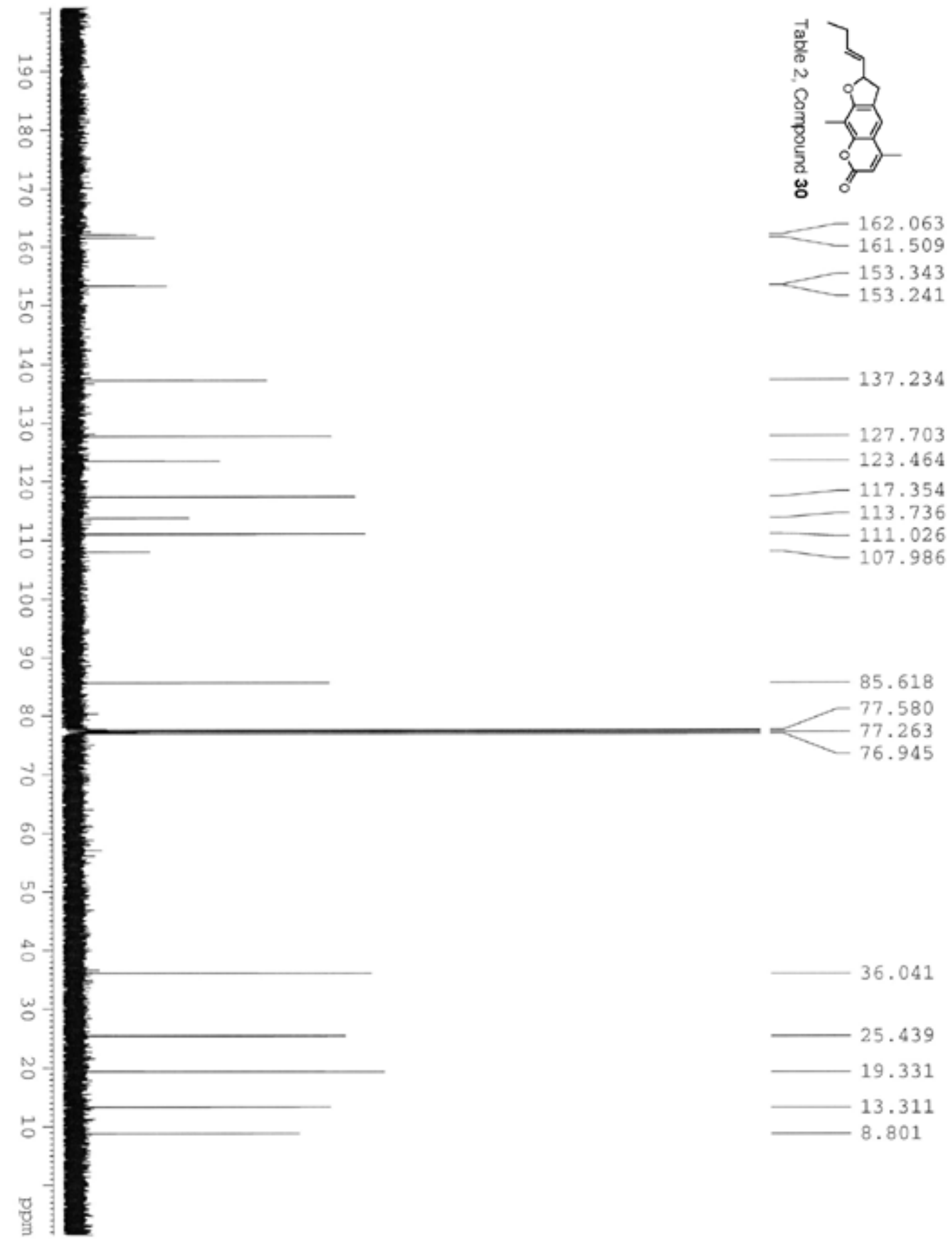



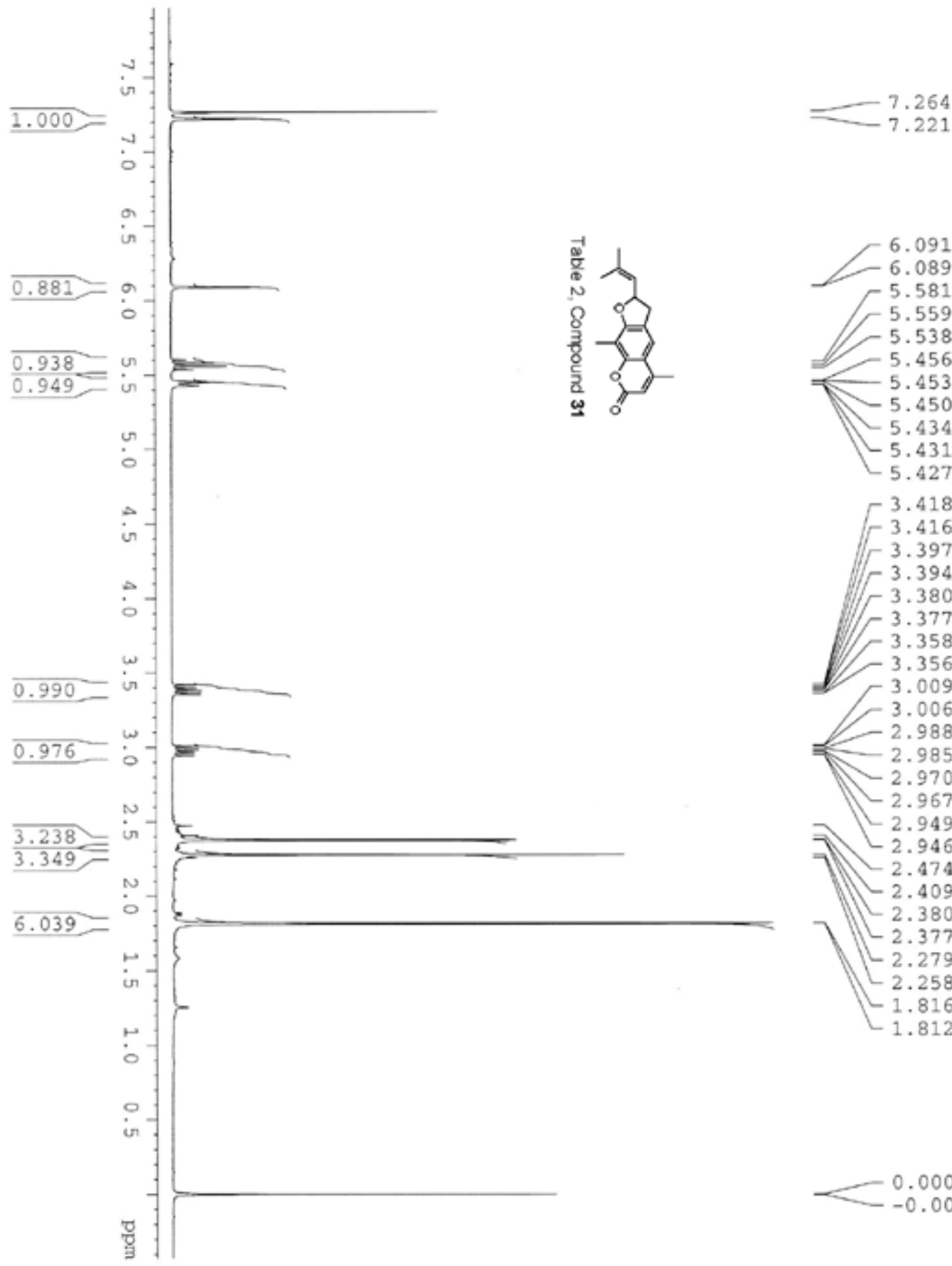

- 3.418

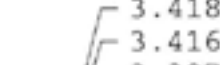

- 3.397

II -3.394

3.380
3.377

$-3.377$

$-3.358$

$-3.356$

$-3.009$

$-3.006$

-2.988
-2.985

- 2.985

$-2.970$

-2.967
2.949

7 $\llcorner 2.946$

$\longrightarrow 2.474$

-2.409
-2.380

$-2.380$

$-2.377$

$\backslash 2.279$

$-2.258$

ᄂ 1.812

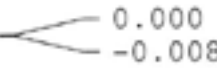




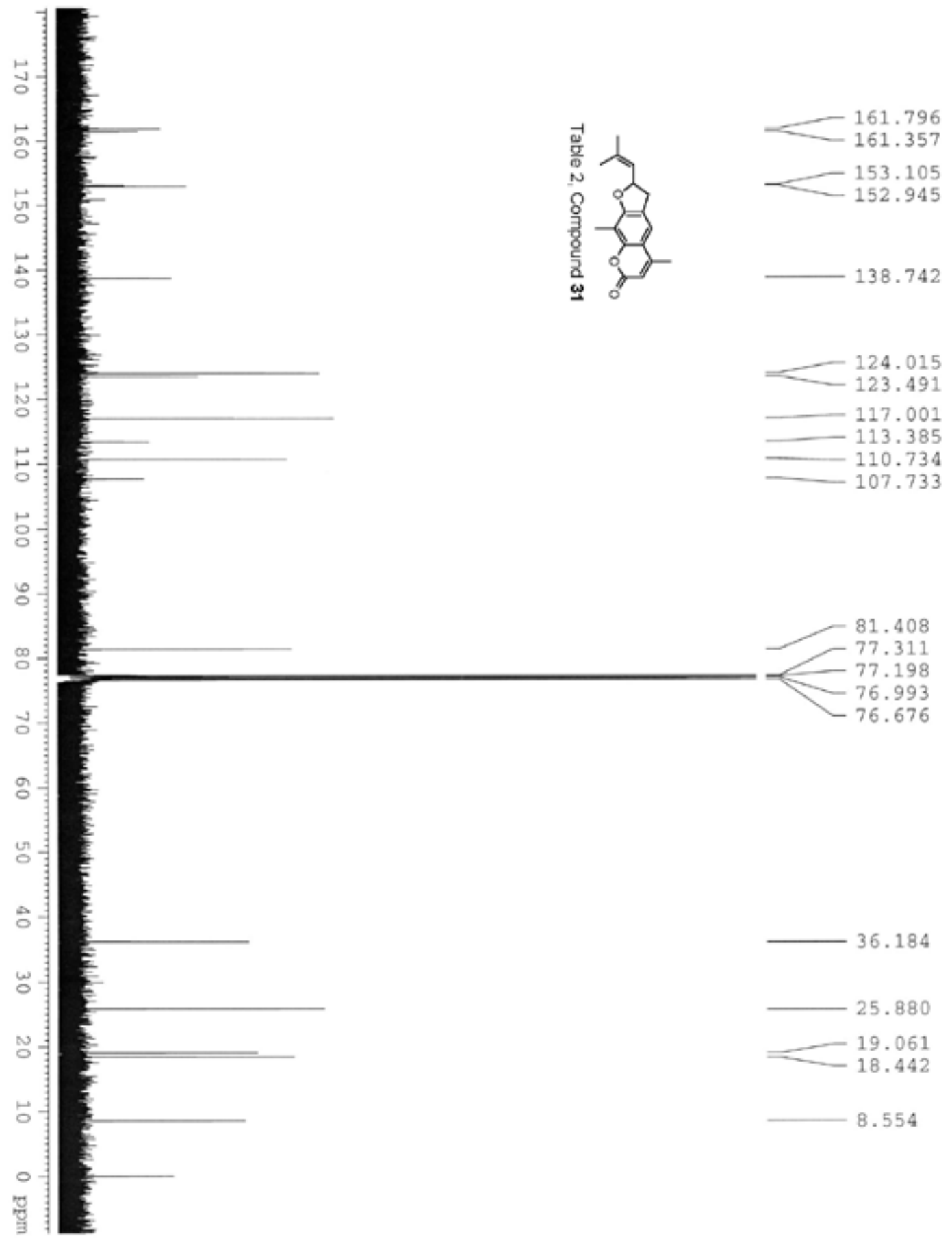




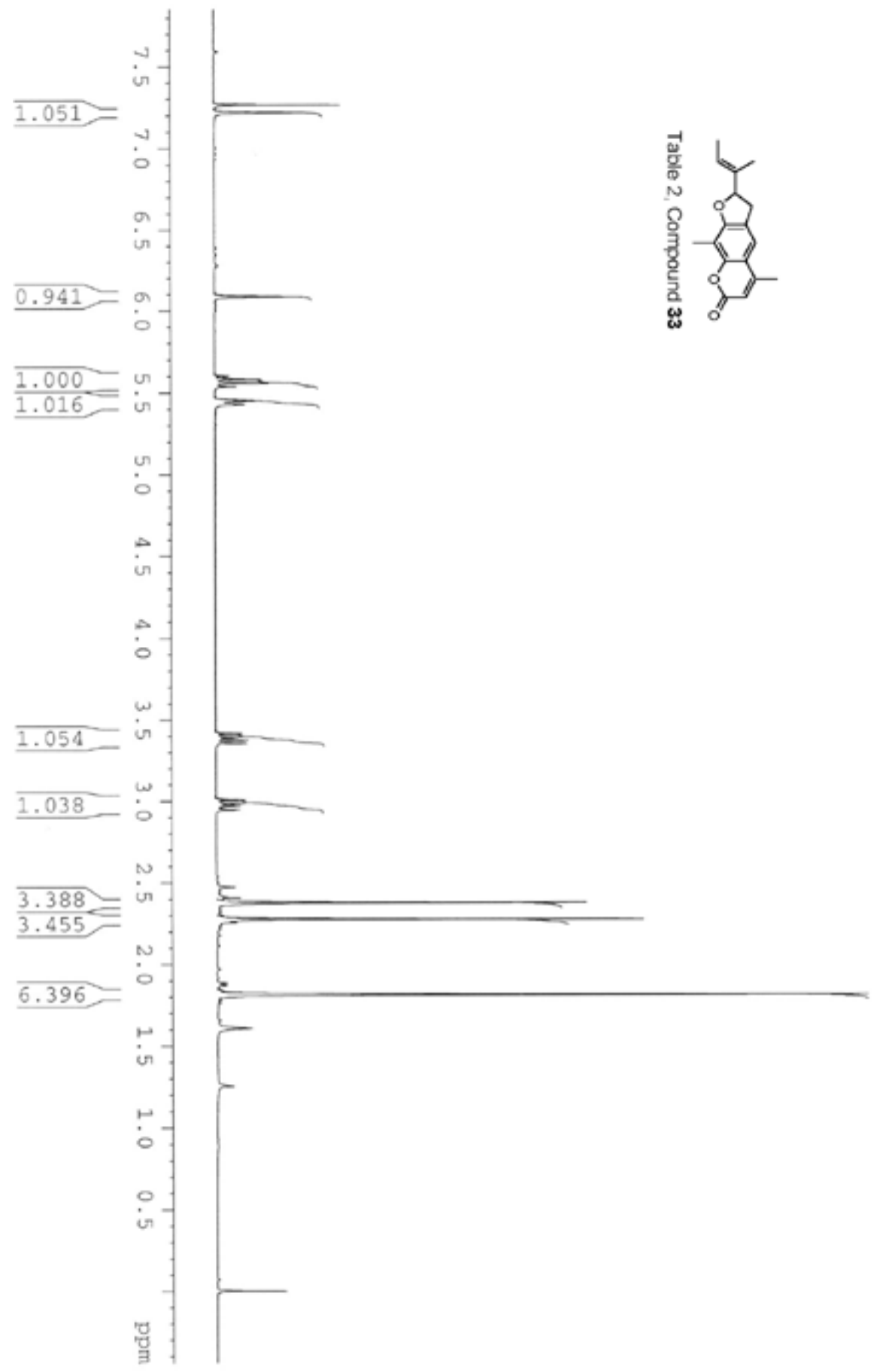

-7.266
-7.220

6.089
-6.086

5.603

$-5.581$

$-5.559$

$-5.538$

$-5.456$

T 5.452

$-5.449$

$-5.433$

$-5.430$

$\llcorner 5.427$

$\Gamma^{3.418}$

T 3.380

$-3.358$

$-3.008$

$\sqrt{-3.005}$

$/ / 2.987$

$-2.984$

2.970
-2.967

$-2.967$

$-2.949$

$-2.946$

$-2.474$

$-2.471$

-2.409
-2.379

$-2.379$

$-2.376$

$-2.278$

$\lcm{-2.257}$

$-1.815$

$\checkmark 1.812$

$-1.608$

$-1.252$

0.000 


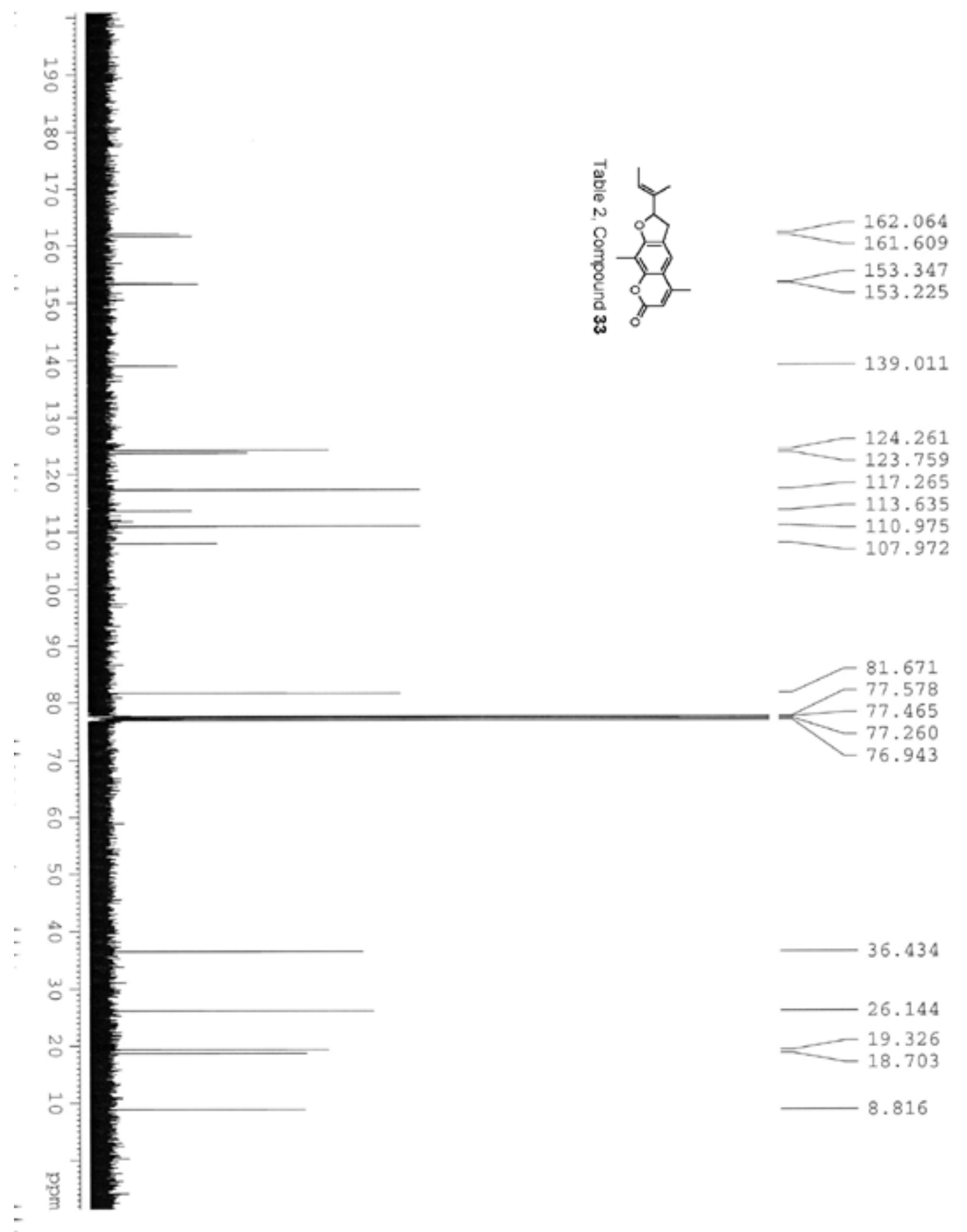



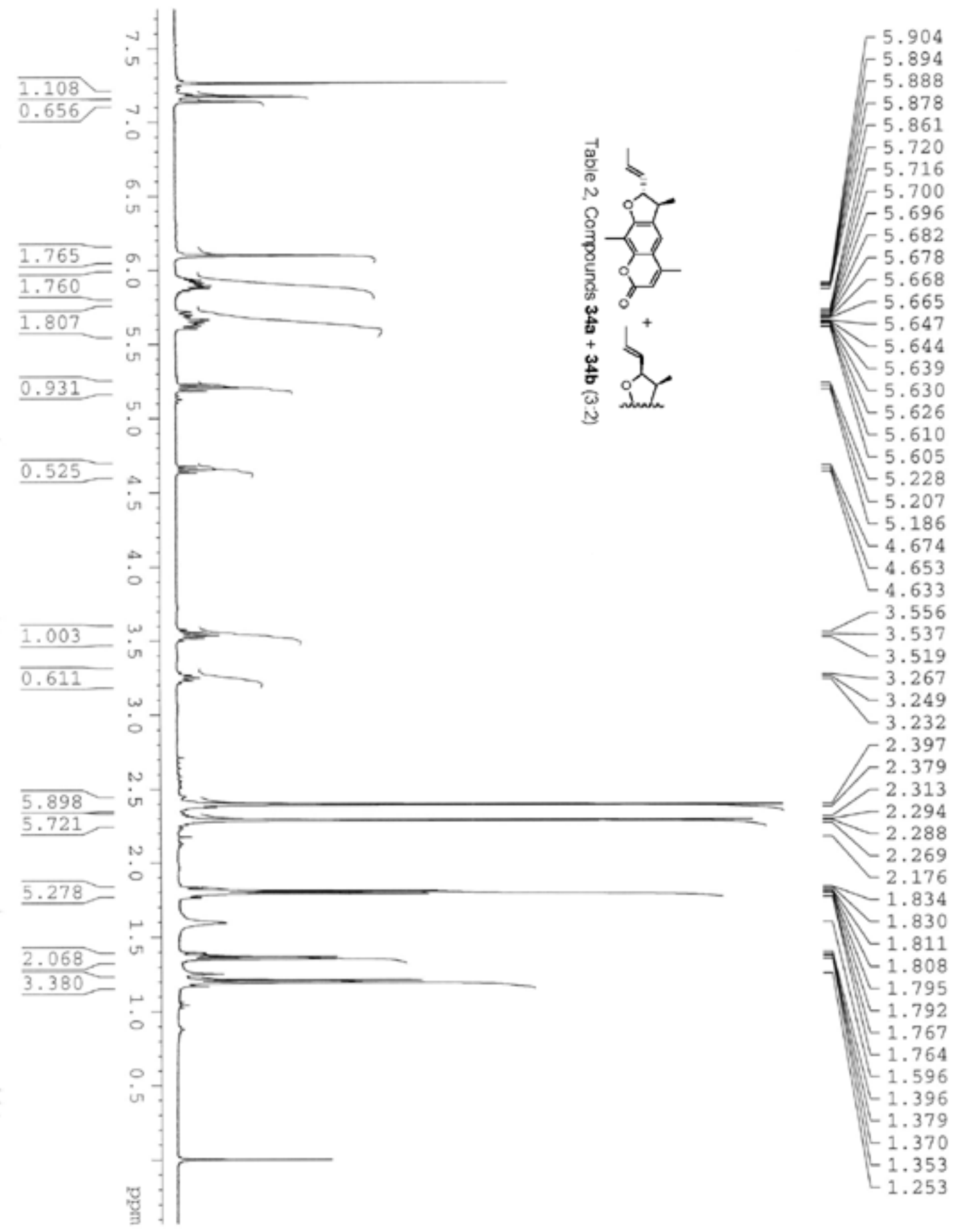


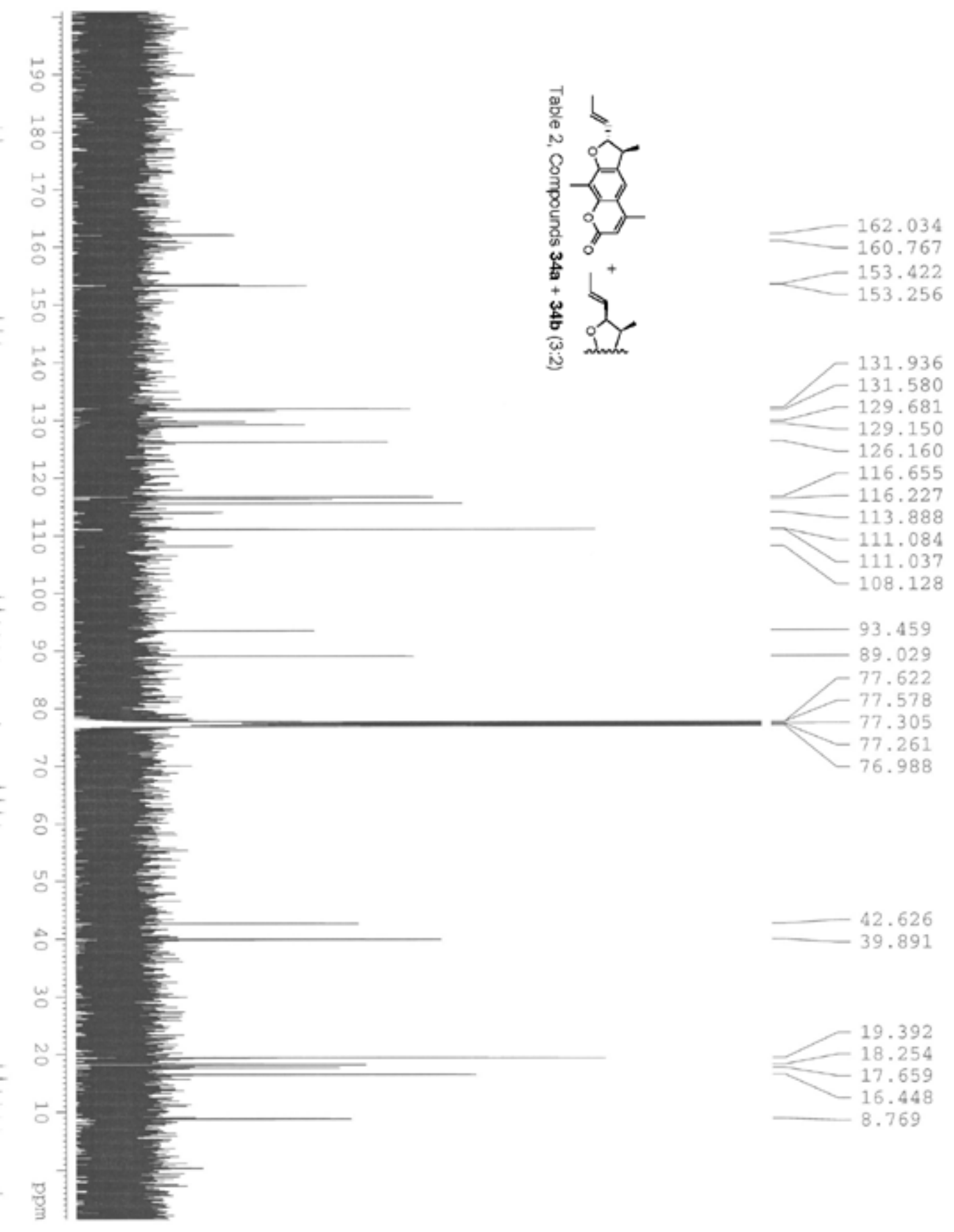




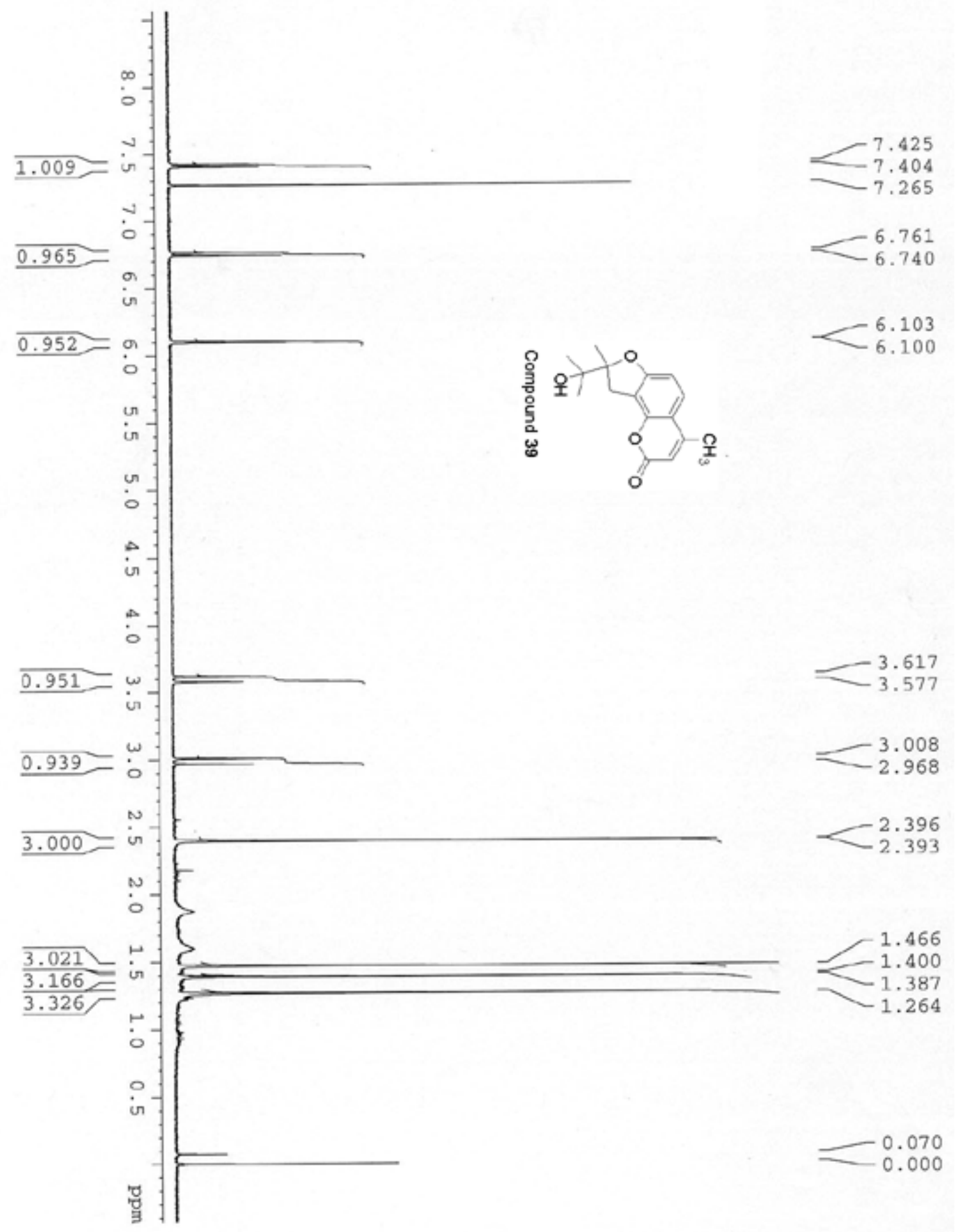



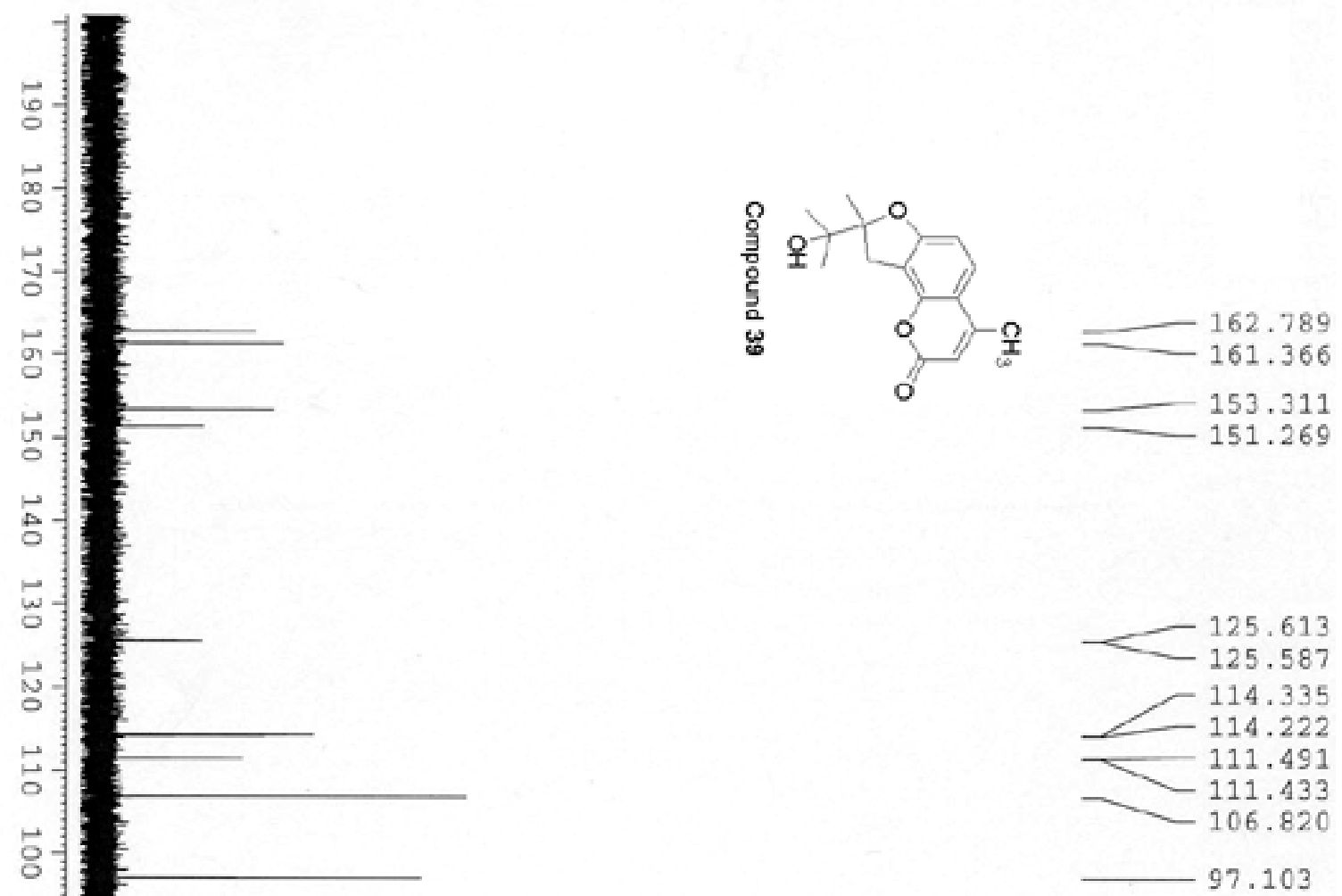

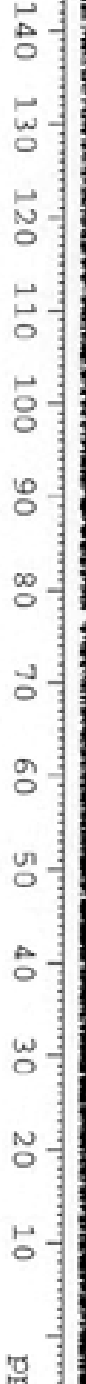

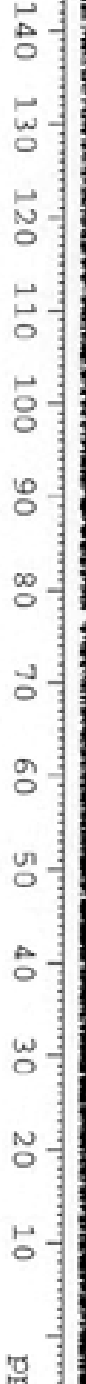

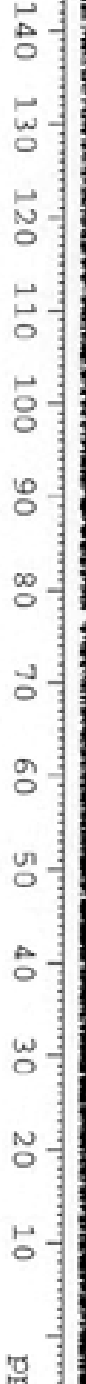

s
$\circ$
0
$\circ$
$\circ$
$\circ$
$\circ$
$\circ$
$\circ$
$\circ$
$\circ$
$\circ$
$\circ$
$\circ$
$\circ$

s
$\circ$
0
$\circ$
$\circ$
$\circ$
$\circ$
$\circ$
$\circ$
$\circ$
$\circ$
$\circ$
$\circ$
$\circ$
$\circ$

s
0
0
0
$\circ$
$\circ$
$\circ$
$\circ$
$\circ$
$\circ$
$\circ$
$\circ$
$\circ$
$\circ$
$\circ$

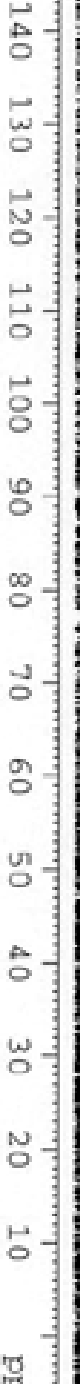

"ृ

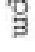

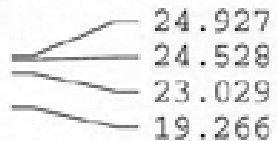



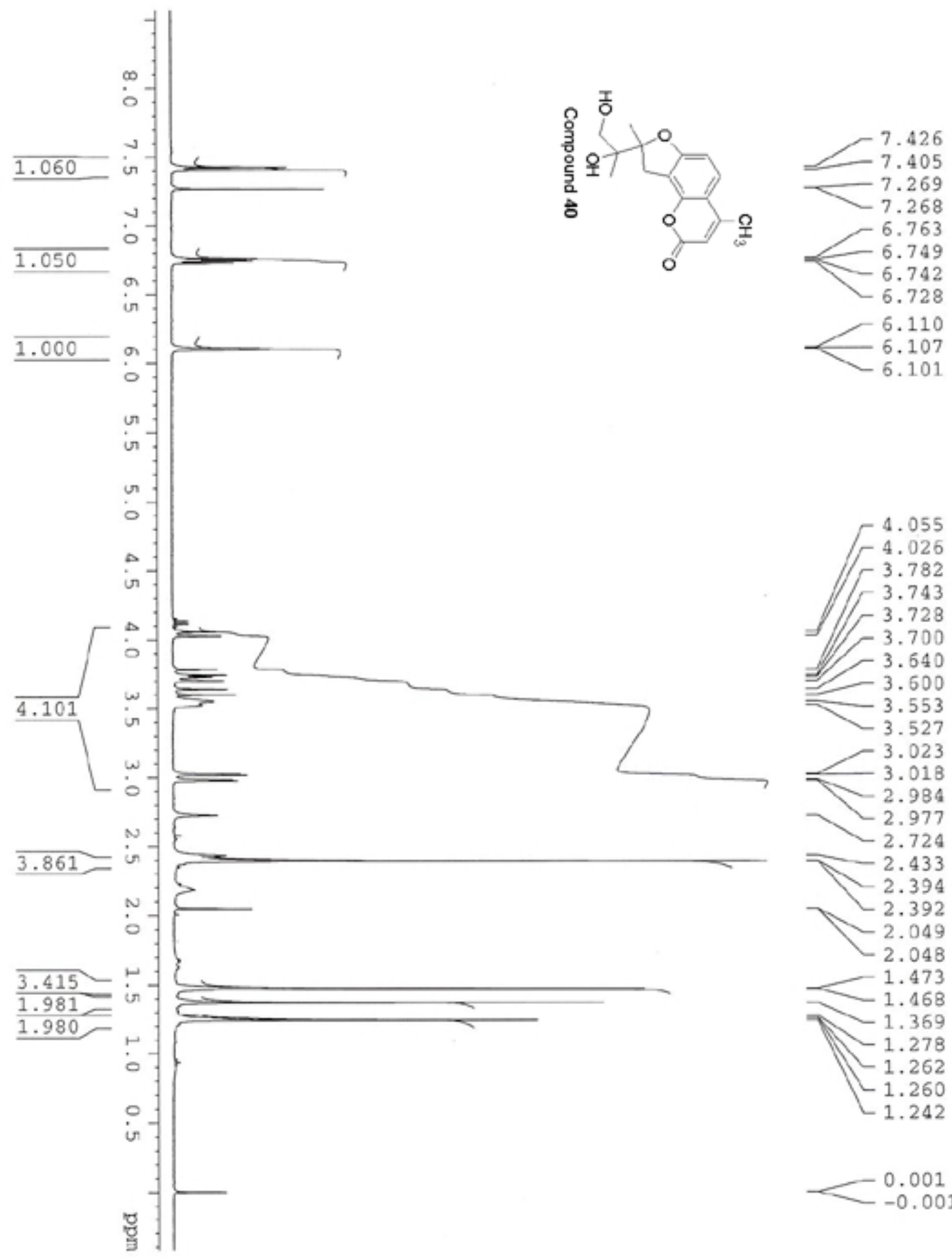

$F^{4.026}$

3.782
3.743

3.743
3.723

$\Gamma^{3.728}$

$-3.700$

$\begin{array}{r}3.700 \\ -3.640 \\ -3.503 \\ \hline\end{array}$

3.553

$-3.527$

3.023
-3.018
-2.984

$\checkmark 2.977$

$-2.724$

$=2.433$

$\begin{array}{r}2.394 \\ -2.392 \\ \hline\end{array}$

$\sim 2.049$

$-2.048$

1.473

$-1.468$

$\longrightarrow 1.369$

$-1.278$

$-1.262$

$-1.260$

$-1.242$

$-0.001$

$\chi_{-0.001}$ 

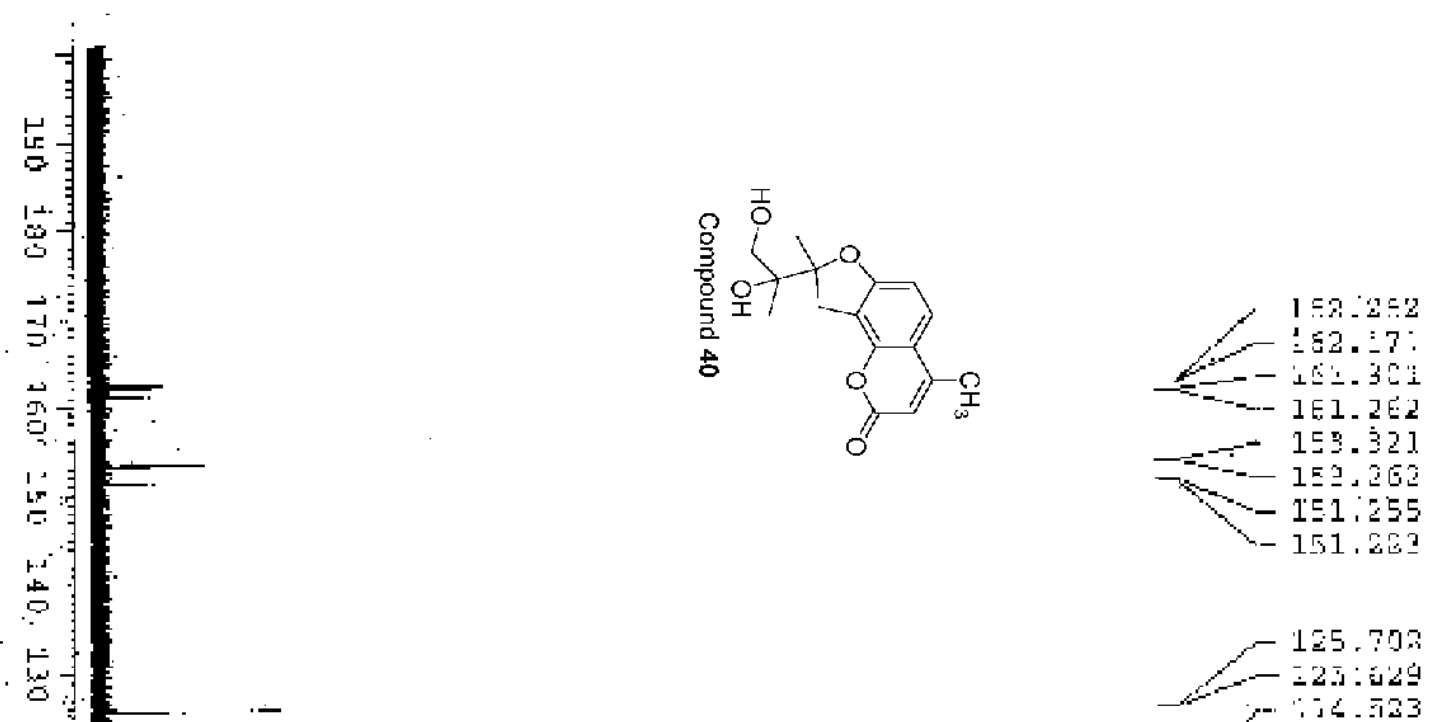

F 1]2.75

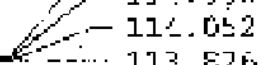

7.--111,696

$-3-111.544$

它

$\$$

3
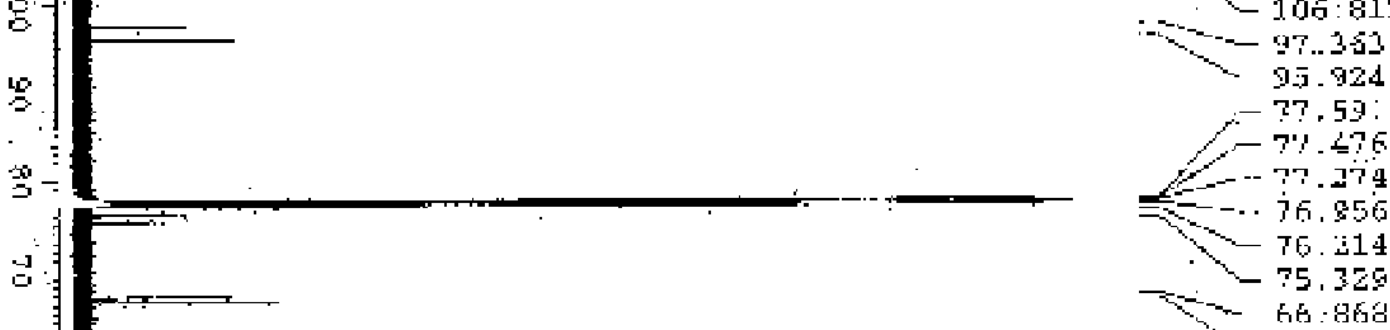

49.924

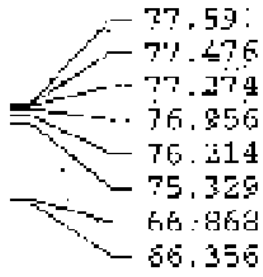

$\$$

列

䛼录

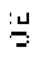

$\therefore$

.

5

思

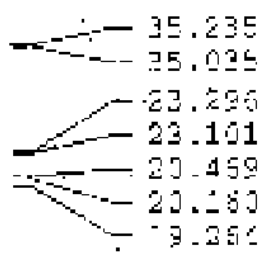




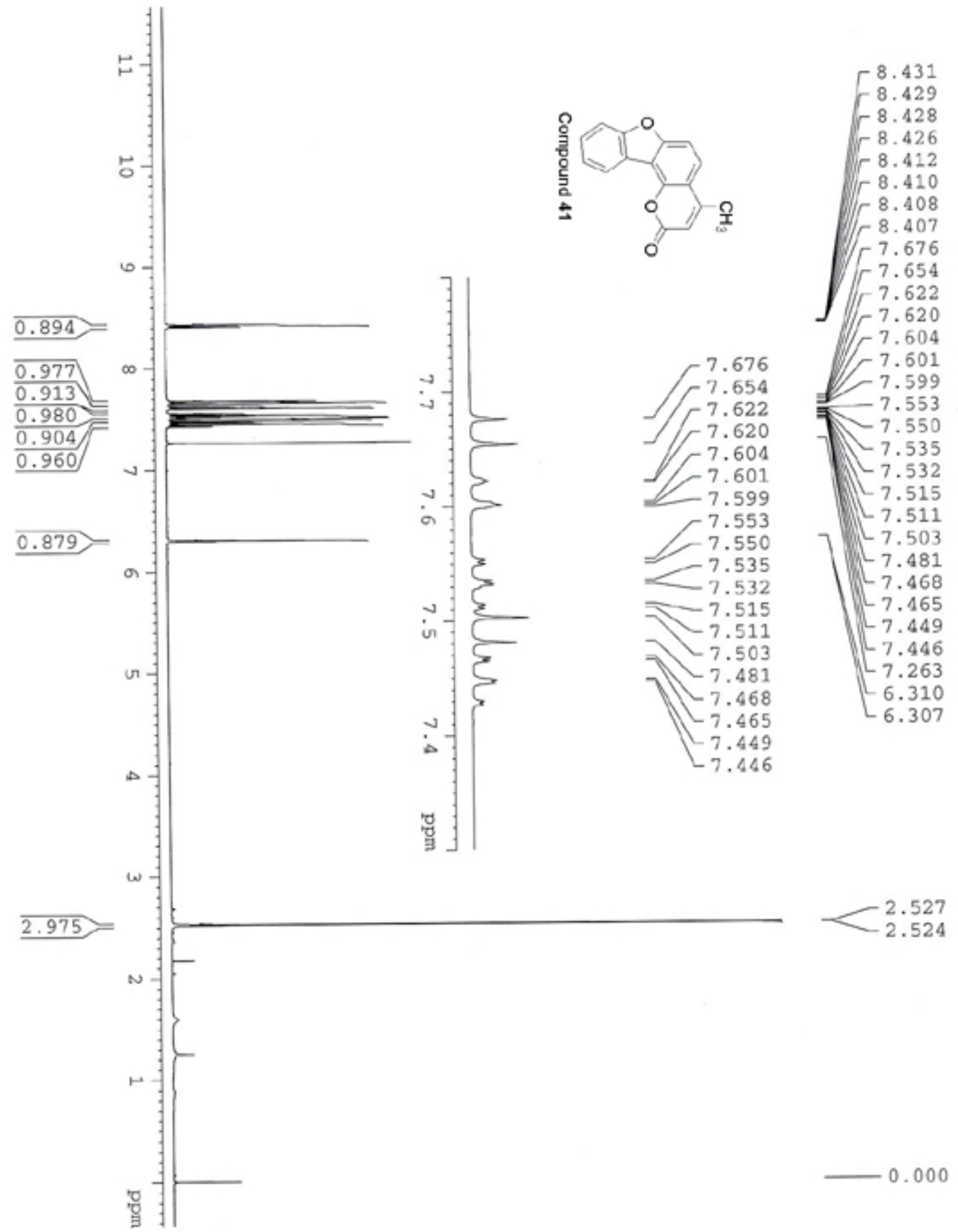



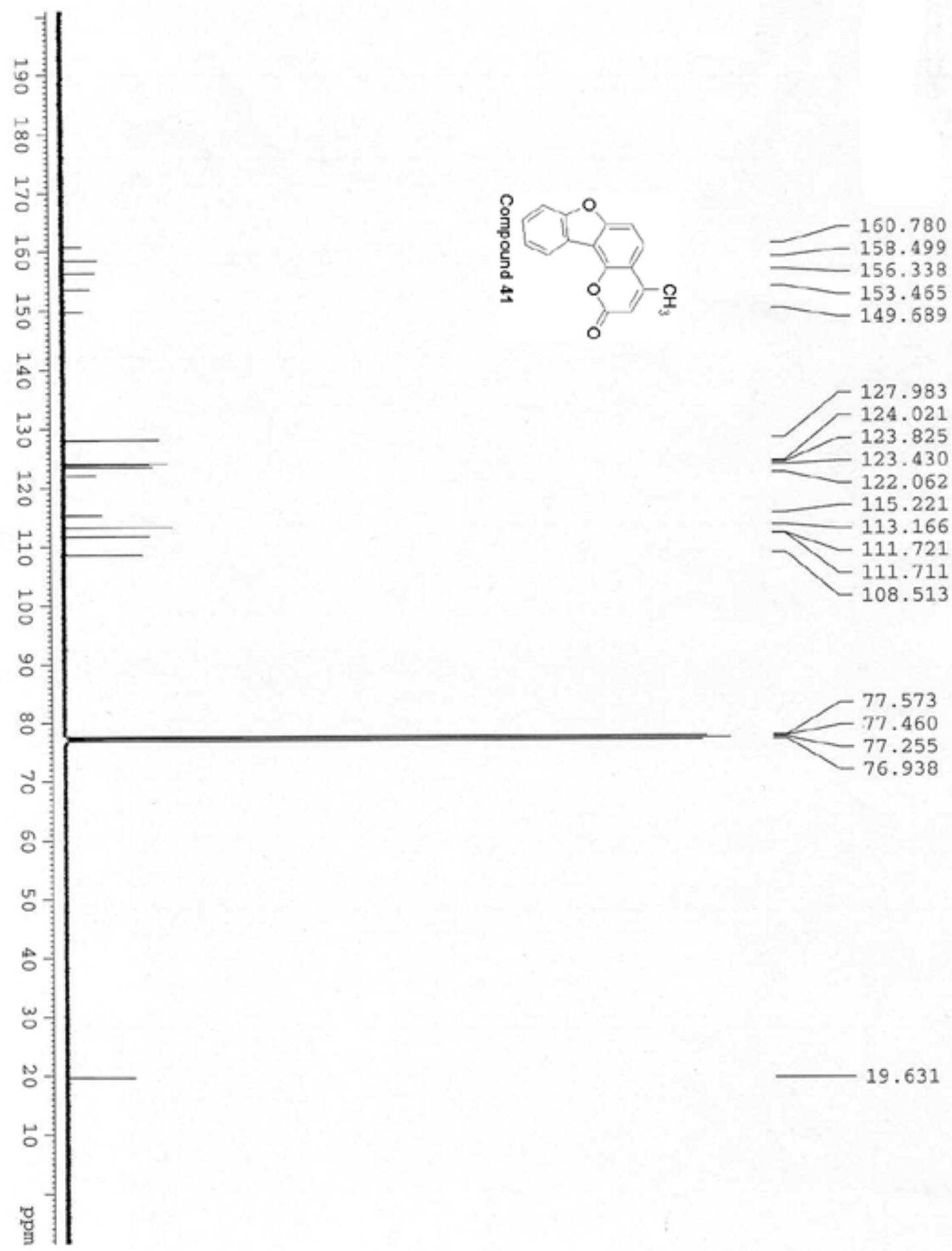